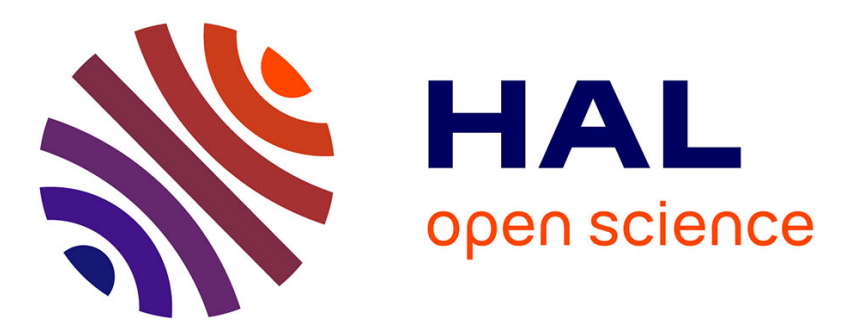

\title{
Reactivity of N-protected 5-(2-bromophenyl)tetrazoles in palladium-catalyzed direct arylation of heteroarenes or fluorobenzenes
}

Sabah Chikhi, Safia Djebbar, Jean-François Soulé, Henri Doucet

\section{- To cite this version:}

Sabah Chikhi, Safia Djebbar, Jean-François Soulé, Henri Doucet. Reactivity of N-protected 5-(2bromophenyl)tetrazoles in palladium-catalyzed direct arylation of heteroarenes or fluorobenzenes. Journal of Organometallic Chemistry, 2017, 831, pp.55-63. 10.1016/j.jorganchem.2016.12.026 . hal01475812

HAL Id: hal-01475812

https://hal-univ-rennes1.archives-ouvertes.fr/hal-01475812

Submitted on 11 Apr 2017

HAL is a multi-disciplinary open access archive for the deposit and dissemination of scientific research documents, whether they are published or not. The documents may come from teaching and research institutions in France or abroad, or from public or private research centers.
L'archive ouverte pluridisciplinaire HAL, est destinée au dépôt et à la diffusion de documents scientifiques de niveau recherche, publiés ou non, émanant des établissements d'enseignement et de recherche français ou étrangers, des laboratoires publics ou privés. 


\title{
Reactivity of $N$-Protected 5-(2- Bromophenyl)tetrazoles in Palladium-Catalyzed Direct Arylation of Heteroarenes or Fluorobenzenes
}

\author{
Sabah Chikhi, ${ }^{a, b}$ Safia Djebbar, ${ }^{b}$ Jean-François Soulé, ${ }^{a} *$ Henri Doucet ${ }^{a}$.
}

\begin{abstract}
a UMR 6226 CNRS-Université de Rennes 1 "Organométalliques, Matériaux et Catalyse", Campus de Beaulieu, 35042 Rennes, France.

${ }^{\mathrm{b}}$ Laboratoire d'Hydrométallurgie et Chimie Inorganique Moléculaire, Faculté de Chimie U.S.T.H.B. Bab-Ezzouar, Alger, Algeria.
\end{abstract}

KEYWORDS. Palladium, Heterocycles, Aryl Bromides, Tetrazoles, Catalysis, $\mathrm{C}-\mathrm{H}$ Activation.

\begin{abstract}
A new route allowing the one-step synthesis of (2-heteroarylphenyl)tetrazole and fluorinated biphenyltetrazole derivatives is disclosed. By using $2 \mathrm{~mol} \%$ of an air-stable diphosphinepalladium catalyst $\left[\mathrm{PdCl}\left(\mathrm{C}_{3} \mathrm{H}_{5}\right)(\mathrm{dppb})\right]$, potassium pivalate as base and dimethylacetamide as solvent, a wide range of heteroarenes (e.g., thiazoles, (benzo)thiophenes, furans, pyrroles, and imidazo[1,2a]pyridine) and polyfluorobenzenes was easily coupled with $N$-protected (2-bromophenyl)tetrazoles in high yields.
\end{abstract}

\section{INTRODUCTION}

Angiotensin II receptor blockers (ARBs) are an important class of medicinal drugs, which modulate the renin-angiotensin system, prescribed to regulate the high blood pressure, or used in case of diabetic nephropathy or against congestive heart failure. Nonpeptide ARBs based on a biphenyltetrazole motif, such as Losartan and Candesartan, have emerged as highly effective antihypertensives, which could be orally administered (Figure 1). ${ }^{[1]}$ Embusartan is an analogue containing a fluorinated biphenyl unit, ${ }^{[2]}$ and Zolarsartan contains an heteroaromatic ring (benzofuran) as spacer (Figure 1). ${ }^{[3]}$ Due to their wide use and relatively high cost, the discovery of new routes for the synthesis of ARBs represents a major socio-economic challenge for pharmaceutical companies and an important challenge for academic research groups. 

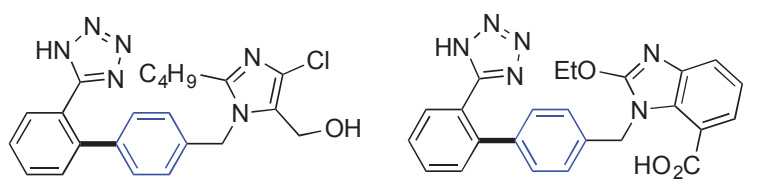

Losartan
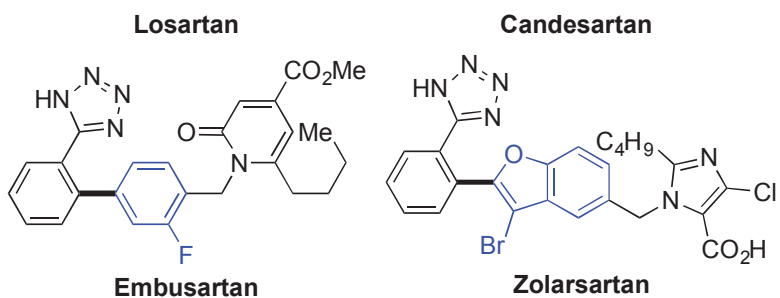

Figure 1. Structure of Angiotensin II Receptor Blockers

One of the most widespread disconnections of the 5-biphenyl tetrazole motif involves the $\mathrm{C}-$ $\mathrm{C}$ bond between the two phenyls of the biphenyl unit. Therefore, palladium-catalyzed crosscoupling reactions using aryl halides in the presence of nucleophilic organometallic or main group element reagents, represent an appropriate approach for their preparation. ${ }^{[4]}$ In recent years, the transition metal-catalyzed direct arylation, via a $\mathrm{C}-\mathrm{H}$ bond activation, has become one of the most sustainable methods for the $\mathrm{C}-\mathrm{C}$ bond formation. ${ }^{[5]}$ Indeed, compared to other classical transition metal-catalyzed reactions such as Stille, Suzuki or Negishi couplings, they do not require the preliminary synthesis of organometallic derivatives, and only HX associated to a base is generated as by-product. In this line, Seki described an elegant approach for the synthesis of 5-biaryltetrazole derivatives involving rutheniumcatalyzed ortho $\mathrm{C}-\mathrm{H}$ bond arylation of $\mathrm{N}$-protected 5-phenyltetrazole in the presence of aryl bromides. ${ }^{[6]}$ The reaction proceeds in high yield using hydrated- $\mathrm{RuCl}_{3}$ associated to $\mathrm{PPh}_{3}$ as catalytic system in the presence of potassium carbonate as base in $\mathrm{N}$-methylpyrrolidone. However, only 4-bromobenzyl acetate has been used as substrate leading to Valsartan in only a few steps. $\left[\mathrm{RuCl}_{2}\left(\mathrm{C}_{6} \mathrm{H}_{6}\right)\right]_{2}$ was also found to be a very efficient catalyst for the $\mathrm{C}-\mathrm{H}$ bond activation/arylation of $\mathrm{N}$-protected 5-phenyltetrazoles and was applied to the synthesis of Losartan. ${ }^{[7]}$ Using the same strategy, but with $\left[\mathrm{RuCl}_{2}(p \text {-cymene })\right]_{2}$ as catalyst, Seki described the environmental-friendly total synthesis of Candesartan Cilexetil. ${ }^{[8]}$ In 2013, Akermann and co-workers demonstrated a significant rate-acceleration by using carboxylates as additives, in ruthenium(II)-catalyzed $\mathrm{C}-\mathrm{H}$ bond arylation of 5-aryltetrazoles. They employed a very broad substrate scope including two heteroaryl bromides (2-bromothiophene and 3bromopyridine). ${ }^{[9]}$ To our knowledge, the reverse $\mathrm{C}-\mathrm{H}$ bond functionalization strategy, namely the use of 5-(2-bromophenyl)tetrazole derivatives and (hetero)arenes has not yet been described, although it could open a new route to the straightforward synthesis of novel (2heteroarylphenyl)tetrazole and fluorinated biphenyltetrazole analogues. From this consideration, we decided to explore the reactivity of different $\mathrm{N}$-protected 5-(2bromophenyl)tetrazoles in the palladium-catalyzed direct arylation in the presence of a wide range of heteroarenes and fluorobenzenes.

\section{RESULTS AND DISCUSSION}

First, the reaction between 5-(2-bromophenyl)tetrazole and 2-ethyl-4-methylthiazole was attempted in the presence of $2 \mathrm{~mol} \%$ of $\mathrm{Pd}(\mathrm{OAc})_{2}$ or $\mathrm{PdCl}\left(\mathrm{C}_{3} \mathrm{H}_{5}\right)(\mathrm{dppb})$ as catalysts associated to 2 equivalents of potassium acetate (KOAc) in DMA. However, no formation of the desired coupling product was detected. We attributed this lack of reactivity to the free $\mathrm{NH}$ of the tetrazole moiety, which might poison the palladium catalyst by coordination. Therefore, we decided to protect the tetrazole using different protecting group. 5-(2Bromophenyl)tetrazole was treated by 2 equivalents of sodium hydride in DMF, then benzyl 
bromide, pivaloyl chloride, or methyl iodide were introduced to furnish the desired $\mathrm{N}$ protected 5-(2-bromophenyl)tetrazoles 1a-1c in good yields (Scheme 1).

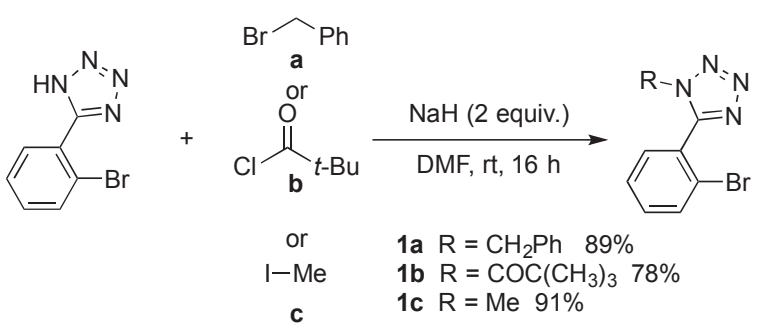

Scheme 1. Protection of 5-(2-Bromophenyl)tetrazole.

Having the $N$-protected 5-(2-bromophenyl)tetrazoles 1a-1c in hand, we next evaluated their reactivities in Pd-catalyzed direct arylation with a set of (hetero)arenes. N-benzyl-5-(2bromophenyl)tetrazole 1a was firstly used as aryltetrazole source (Scheme 2). The arylation of 2-ethyl-4-methylthiazole occurred at $\mathrm{C} 5$ position in the presence of $2 \mathrm{~mol} \%$ of $\mathrm{PdCl}\left(\mathrm{C}_{3} \mathrm{H}_{5}\right)(\mathrm{dppb})$ associated to 2 equivalents of PivOK in DMA to allow the synthesis of 2 in $75 \%$ yield. It is important to note that the use of $\mathrm{KOAc}$ as base or $\mathrm{Pd}(\mathrm{OAc})_{2}$ as catalyst was less effective than the use of $\mathrm{PivOK}$ or of $\mathrm{PdCl}\left(\mathrm{C}_{3} \mathrm{H}_{5}\right)(\mathrm{dppb})$, as under these conditions, 2 was obtained in only $12 \%$ or $34 \%$ yields, respectively. Using $2 \mathrm{~mol} \% \operatorname{PdCl}\left(\mathrm{C}_{3} \mathrm{H}_{5}\right)(\mathrm{dppb})$, PivOK in DMA as reactions conditions, 2-isobutylthiazole was also arylated at $\mathrm{C} 5$ position to give the coupling product 3 in $79 \%$ yield. Then, we turned our attention to the formation of 5-(2-(thiophen-2-yl)phenyl)tetrazole, which is also an important motif, as some of these analogues display biological properties in regulation of blood pressure acting on the Angiotensin II receptors. ${ }^{[10]}$ 2-Methylthiophene, 2-acetyl-3-methylthiophene or benzothiophene were arylated at their $\mathrm{C} 5$ or $\mathrm{C} 2$ positions to allow the formation of phenyl(benzo)thiophene analogues 4-6 in 69-74\% yields. Furans are also suitable coupling partners. For examples, 2-n-butylfuran and 2-butanoylfuran in the presence of $N$-benzyl-5-(2bromophenyl)tetrazole 1a were arylated at C5 position to deliver the 5-(2-(furan-2yl)phenyl)tetrazole derivatives 7 and $\mathbf{8}$ in $82 \%$ and $88 \%$ yields, respectively. $N$ Methylpyrrole was also successfully coupled with 1a, but 4 equivalents of this reactant were used to prevent the 2,5-diarylation of pyrrole, affording selectively the C2-arylated product 9 in an excellent yield. Imidazo[1,2-a]pyridine is an heterocycle present in many pharmaceutical products. N-Benzyl-5-(2-bromophenyl)tetrazole 1a smoothly reacted with imidazo[1,2-a]pyridine to afford $\mathbf{1 0}$ in $\mathbf{9 8 \%}$ yield. Polyfluorinated molecules are ubiquitous in medicinal chemistry, owing to fluorine atom properties (i.e., electronegativity, size, lipophilicity, and electrostatic interactions), which induce a dramatic change in the molecules behavior. However, the introduction of fluorine atoms at specific positions of a molecule remains challenging. Alternatively, the use of starting materials containing fluorine atoms in palladium-catalyzed $\mathrm{C}-\mathrm{H}$ bond arylations offers straightforward routes to (poly)fluorinated molecules. ${ }^{[11]}$ We used similar reaction conditions to those we had previously described for the direct arylation of fluorobenzene derivatives. ${ }^{[11 \mathrm{~b}]}$ Such conditions promoted the coupling of pentafluorobenzene with 1a allowing the formation of $\mathbf{1 1}$ in $75 \%$ yield. The reaction was also performed with 3,5-difluorobenzonitrile, and the single regioisomer $\mathbf{1 2}$ was isolated in $67 \%$ yield. As expected, the arylation took place at the position flanked by the two fluorine atoms, which is known to be the most reactive site under concerted metalation-deprotonation mechanism. ${ }^{[12]}$ It is important to note that, after benzyl deprotection and nitrile reduction into an amino group, the derivative $\mathbf{1 2}$ could represent an intermediate for the preparation of fluorinated analogues of Losartan, Candesartan, or Embusratan (c.f., Figure 1). 

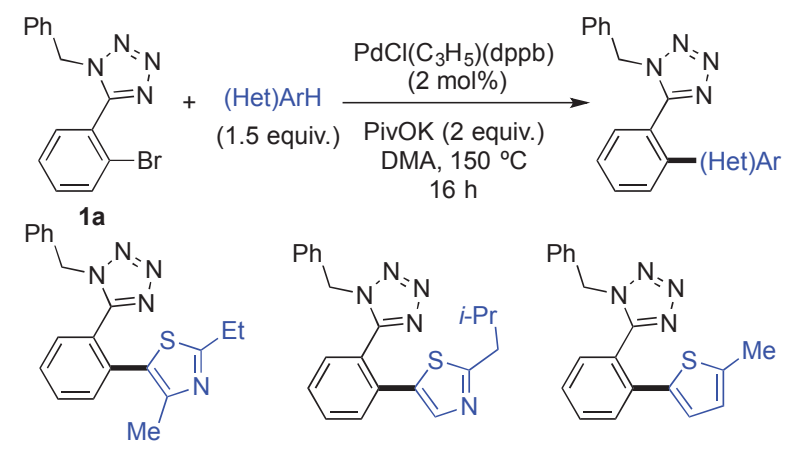

$275 \%$

$(12 \%)^{[a]}(34 \%)^{[b]}$

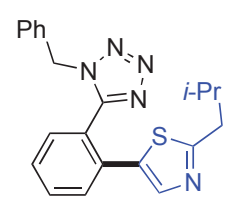

$379 \%$

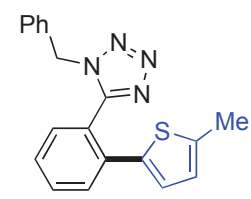

$472 \%$

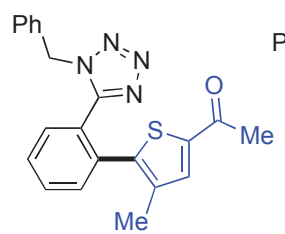

$574 \%$

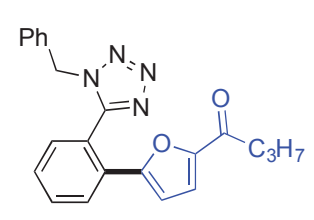

$888 \%$

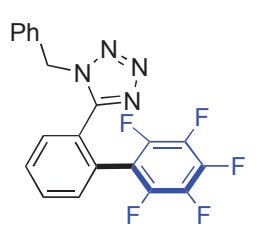

$1175 \%$

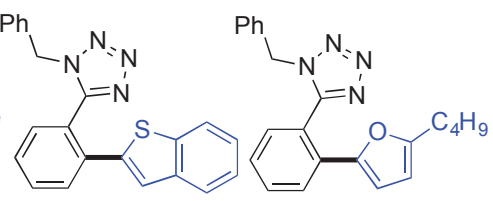

6 69\%

$L_{N}{ }^{N}=N$

$782 \%$

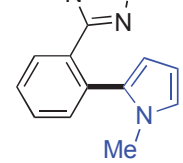

9 92\%[c]

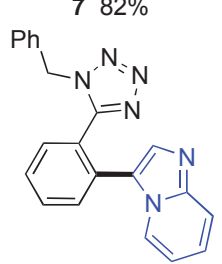

$1098 \%$

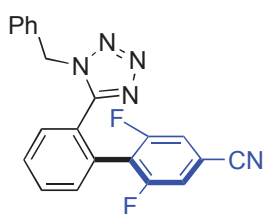

$1267 \%$

Scheme 2. Scope of (Hetero)Arenes in Pd-Catalyzed direct Arylation of 1a. [a] $\mathrm{Pd}(\mathrm{OAc})_{2}(2$ mol\%) was used instead of $\mathrm{PdCl}\left(\mathrm{C}_{3} \mathrm{H}_{5}\right)(\mathrm{dppb})$. [b] KOAc was used instead of PivOK. [c] 4 Equiv. of $N$-methylpyrrole were used.

Next, we investigated the reactivity of 5-(2-bromophenyl)-1-pivalyltetrazole $\mathbf{1 b}$ as aryl source in Pd-catalyzed direct arylation of a set of heteroarenes and polyfluorobenzenes (Scheme 3). In contrast to the benzyl protecting group that can be removed by Pd-catalyzed hydrogenolysis, ${ }^{[13]}$ pivaloyl protected tetrazoles can be deprotected under reductive conditions. $^{[14]}$ In almost all cases, higher yields were obtained with the pivaloyl tetrazole $\mathbf{1 b}$ than with 1a, which bears a benzyl as protecting group. This higher reactivity might be explained by the electron withdrawing character of the pivaloyl group. Indeed, thiazole derivatives were easily coupled with $\mathbf{1 b}$, affording $\mathbf{1 3}$ and $\mathbf{1 4}$ in $88 \%$ and $91 \%$ yields, respectively. 2-Methylthiophene, 2-acetyl-4-methylthiophene and benzothiophene also displayed high reactivities, as the desired products 15-17 were isolated in very high yields. The $N$-protected 5-(2-(furan-2-yl)phenyl)tetrazoles 18 and 19 were obtained in 95\% and 97\% yields from 2- $n$-butylfuran and 2-butyrylfuran, respectively. Again, $N$-methylpyrrole was found to be a suitable coupling partner, albeit 4 equivalents were required to afford $\mathbf{2 0}$ in an excellent yield, without formation of the 2,5-diarylated pyrrole. Fluorinated biphenyls were also synthetized by this route. The $\mathrm{C}-\mathrm{H}$ bond activation of 1,2,3,4-tetrafluorobenzene led to 21 in $67 \%$ yield. 

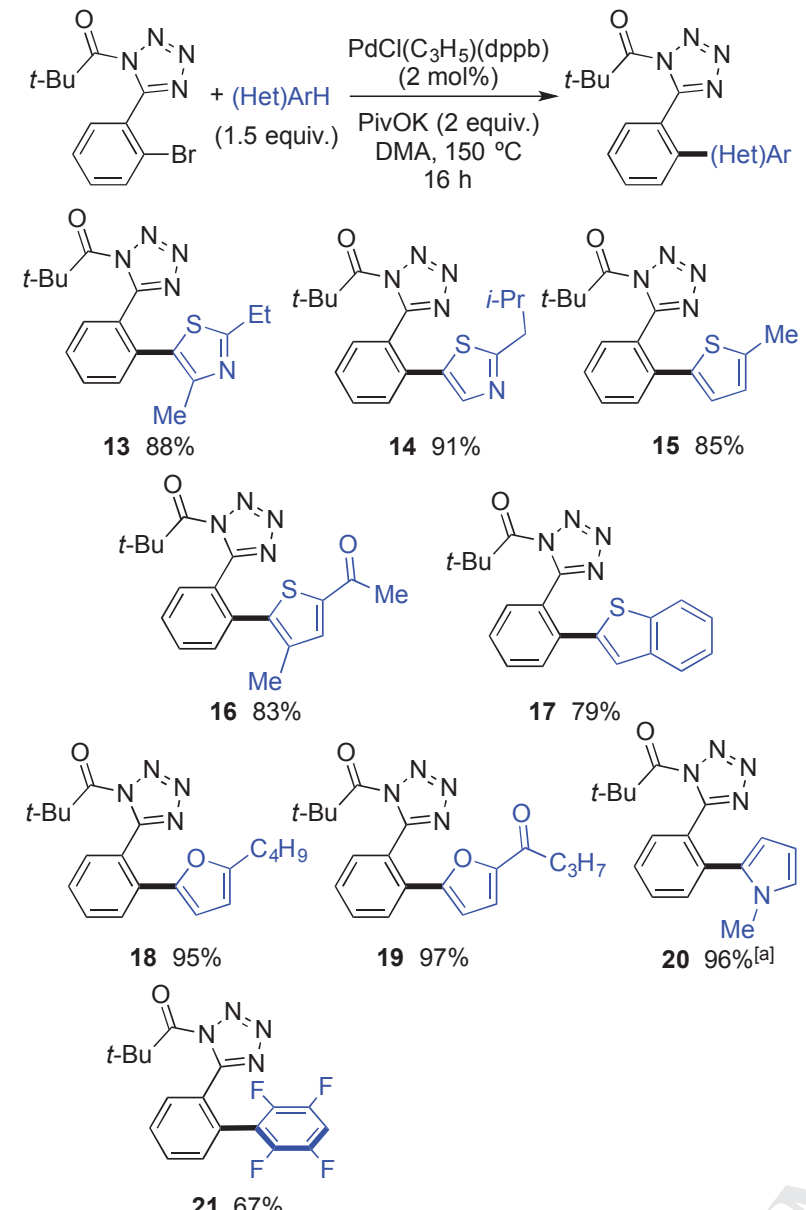

$2096 \%[a]$

Scheme 3. Scope of (hetero)arenes in Pd-catalyzed direct arylation of $\mathbf{1 b}$. [a] 4 Equiv. of $N$ methylpyrrole were used.

Fluorinated biphenyl 21 contains a reactive $\mathrm{C}-\mathrm{H}$ bond in palladium catalysis (i.e., $\mathrm{C}-\mathrm{H}$ bond flanked by two fluorine atoms). Hence, the direct arylation of this reactive $\mathrm{C}-\mathrm{H}$ bond was attempted using 4-bromobenzonitrile. In the presence of the previous reaction conditions, namely $2 \mathrm{~mol} \% \mathrm{PdCl}\left(\mathrm{C}_{3} \mathrm{H}_{5}\right)(\mathrm{dppb})$ associated to 2 equivalents of PivOK in DMA, the triphenyl derivative 22 was obtained in $63 \%$ yield (Scheme 4). This compound could allow, after reduction of the nitrile group, the formation of analogues of Losartan, which contains a 1,2,3,4-tetrafluorophenyl unit as spacer.

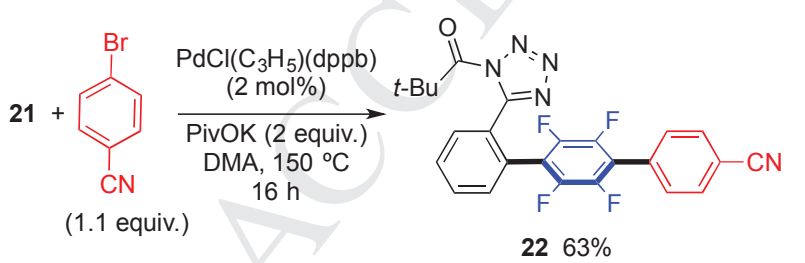

Scheme 4. Direct Arylation of 21.

$N$-Methyl tetrazoles are very important motifs, embedded in many compounds with wide range of applications in materials for energy, ${ }^{[15]}$ bioorganic chemistry, ${ }^{[16]}$ and pharmacology. ${ }^{[1]}$ Therefore, we decided to examine the reactivity of 5-(2-bromophenyl)-1methyltetrazole 1c as coupling partner in the presence of a set of heteroarenes and polyfluorobenzenes, for the efficient synthesis of $N$-methyl 5-((hetero)biphenyl-2-yl)-tetrazole derivatives (Scheme 5). Again, thiazoles were arylated at C5 position allowing the formation 
of the 5-(2-(thiazol-5-yl)phenyl)tetrazole $\mathbf{2 3}$ and $\mathbf{2 4}$ in $89 \%$ and $93 \%$ yields. 2- $n$ Pentylthiophene also displayed a high reactivity in such couplings, as C5-arylated thiophene 25 was isolated in $81 \%$ yield. Furans such as 2-methylfuran and 1-(furan-2-yl)propan-2-one were smoothly arylated at the $\mathrm{C} 5$ position with $\mathbf{1 c}$ to deliver the coupling products $\mathbf{2 6}$ and $\mathbf{2 7}$ in $90 \%$ and $86 \%$ yields, respectively. It is important to note that the acidic $\mathrm{sp}^{3}-\mathrm{C}-\mathrm{H}$ bonds at ortho position of ketone function of 1-(furan-2-yl)propan-2-one was not reactive under these reaction conditions, as no other coupling product than $\mathbf{2 7}$ was detected in the crude mixture. Again the reaction between 1c and 4 equivalents of $N$-methylpyrrole afforded C2-arylated pyrrole 28 in an excellent yield of 93\%. Finally, the synthesis of 5-([biphenyl]-2-yl)-1methyltetrazole was surveyed. From pentafluorobenzene, 1,2,3,4-tetrafluorobenzene and 3,5difluorobenzonitrile, the desired fluorinated biphenyls 29-31 were obtained in 65-70\% yields, through the activation of the $\mathrm{C}-\mathrm{H}$ bond flanked by the two fluorine atoms.

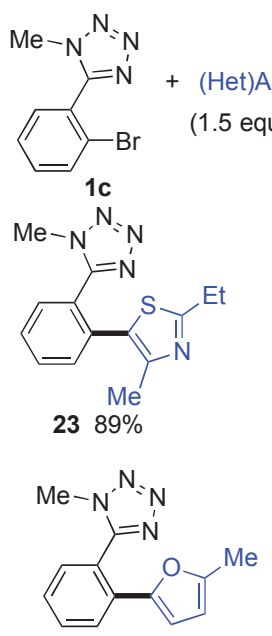

26 90\%

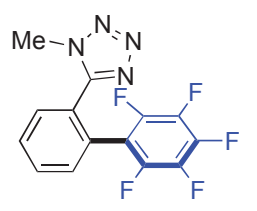

$2969 \%$
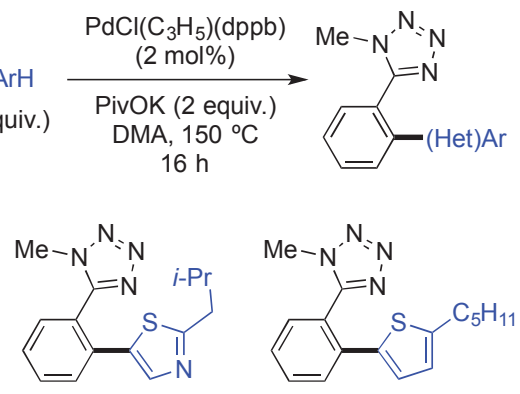

24 93\%

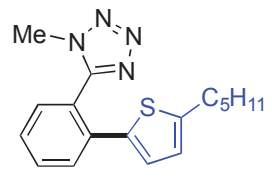

$2581 \%$
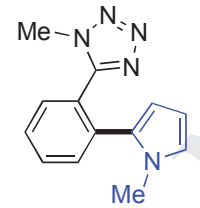

28 93\%[a]

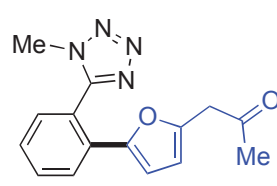

$2786 \%$

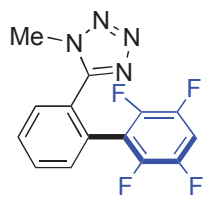

30 65\%

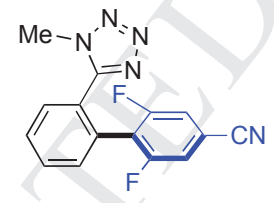

$3170 \%$

Scheme 5. Scope of (hetero)arenes in Pd-catalyzed direct arylation of 1c. [a] 4 Equiv. of $N$ methylpyrrole were used.

\section{CONCLUSION}

In summary, this study demonstrates that $\mathrm{N}$-protected 5-(2-bromophenyl)tetrazoles can be efficiently coupled with a wide variety of heteroarenes (e.g., thiazoles, (benzo)thiophenes, furans, $N$-methylpyrrole, imidazo[1,2-a]pyridine) under $\mathrm{PdCl}\left(\mathrm{C}_{3} \mathrm{H}_{5}\right)(\mathrm{dppb})$-catalyzed $\mathrm{C}-\mathrm{H}$ bond arylation procedure. This procedure which employs an air-stable catalyst and an inexpensive base is efficient, rapid and environmentally attractive. The major by-products are pivalic acid and potassium bromide, instead of the metallic salts produced with more classical cross-coupling reactions such as Suzuki, Stille or Negishi couplings. Moreover, the preparation of an organometallic reagent is not required, reducing the number of steps and therefore the amount of waste in the preparation of these molecules. This methodology is an efficient tool for the synthesis of phenyltetrazoles ortho-substituted by heteroarenes or the synthesis of fluorinated biphenyltetrazoles, and as all the products which have been synthetized by this procedure are new, it could attract interest of medicinal chemists. 


\section{EXPERIMENTAL SECTION}

All reactions were carried out under argon atmosphere with standard Schlenk techniques. DMA ( $N, N$-dimethylacetamide) $(99 \%)$ was purchased from Acros. $\left[\mathrm{Pd}\left(\mathrm{C}_{3} \mathrm{H}_{5}\right) \mathrm{Cl}_{2}(56.5 \%)\right.$ and dppb [1,4-bis(diphenylphosphino)butane] (98\%) were purchased from Alfa Aesar. These compounds were not purified before use. ${ }^{1} \mathrm{H}$ NMR spectra were recorded on Bruker GPX $(400$ or $300 \mathrm{MHz})$ spectrometer. Chemical shifts $(\delta)$ were reported in parts per million relative to residual $\mathrm{CDCl}_{3}$ (7.26 ppm (s) ppm for ${ }^{1} \mathrm{H} ; 77.0 \mathrm{ppm}$ for ${ }^{13} \mathrm{C}$ ), constants were reported in Hertz. ${ }^{1} \mathrm{H}$ NMR assignment abbreviations were the following: singlet (s), doublet (d), triplet (t), quartet (q), doublet of doublets (dd), doublet of triplets (dt), and multiplet (m). ${ }^{13} \mathrm{C}$ NMR spectra were recorded at 100 or $75 \mathrm{MHz}$ on the same spectrometer and reported in ppm. All reagents were weighed and handled in air.

Preparation of the $\mathbf{P d C l}(\mathbf{d p p b})\left(\mathbf{C}_{3} \mathbf{H}_{5}\right)$ catalyst: ${ }^{18]}$ An oven-dried $40-\mathrm{mL}$ Schlenk tube equipped with a magnetic stirring bar under argon atmosphere, was charged with $\left[\mathrm{Pd}\left(\mathrm{C}_{3} \mathrm{H}_{5}\right) \mathrm{Cl}\right]_{2}(182 \mathrm{mg}, 0.5 \mathrm{mmol})$ and dppb $(426 \mathrm{mg}, 1 \mathrm{mmol}) .10 \mathrm{~mL}$ of anhydrous dichloromethane were added, then the solution was stirred at room temperature for twenty minutes. The solvent was removed in vacuum. The yellow powder was used without purification. ${ }^{31} \mathrm{P}$ NMR $\left(81 \mathrm{MHz}, \mathrm{CDCl}_{3}\right) \delta(\mathrm{ppm})=19.3(\mathrm{~s})$.

1-Benzyl-5-(2-bromophenyl)tetrazole (1a): To a stirred suspension of $\mathrm{NaH}(60 \%$ in oil, $0.80 \mathrm{~g}, 20 \mathrm{mmol})$ in DMF $(20 \mathrm{~mL})$ was added in small portions 5-(2-bromophenyl)tetrazole $(2.25 \mathrm{~g}, 10 \mathrm{mmol})$ at $0{ }^{\circ} \mathrm{C}$, and the mixture was stirred at RT for about 1 hour until $\mathrm{NaH}$ disappearance. To this sodio tetrazole mixture, benzyl bromide $(2.05 \mathrm{~g}, 12 \mathrm{mmol})$ was added and the resulting mixture was stirred at RT for $12 \mathrm{~h}$. Then, the reaction mixture was quenched with saturated $\mathrm{NH}_{4} \mathrm{Cl}$ solution $(25 \mathrm{~mL})$, diluted with water $(25 \mathrm{~mL})$, and extracted with AcOEt $(3 \times 50 \mathrm{~mL})$. The organic phase was washed with brine $(50 \mathrm{~mL})$, dried $\left(\mathrm{Na}_{2} \mathrm{SO}_{4}\right)$ and filtered. After removal of all volatiles, the residue was purified by flash chromatography on silica gel (pentane-Et $\left.{ }_{2} \mathrm{O}, 85: 15\right)$ to afford $1 \mathrm{a}(2.80 \mathrm{~g}, 89 \%)$ as a white solid $(\mathrm{mp}=45-47$ $\left.{ }^{\circ} \mathrm{C}\right) .{ }^{1} \mathrm{H}$ NMR $\left(300 \mathrm{MHz}, \mathrm{CDCl}_{3}\right) \delta(\mathrm{ppm}) 7.86(\mathrm{dd}, J=1.8$ and $8.0 \mathrm{~Hz}, 1 \mathrm{H}), 7.75(\mathrm{dd}, J=1.2$ and $8.0 \mathrm{~Hz}, 1 \mathrm{H}), 7.50-7.37(\mathrm{~m}, 6 \mathrm{H}), 7.37-7.30(\mathrm{~m}, 1 \mathrm{H}), 5.87(\mathrm{~s}, 2 \mathrm{H}) .{ }^{13} \mathrm{C} \mathrm{NMR}(100 \mathrm{MHz}$, $\left.\mathrm{CDCl}_{3}\right) \delta(\mathrm{ppm}) 164.4,134.1,133.2,131.7,131.2,129.0,129.0,128.6,128.4,127.4,122.1$, 56.9. Elemental analysis: calcd (\%) for $\mathrm{C}_{14} \mathrm{H}_{11} \mathrm{BrN}_{4}$ (315.17): $\mathrm{C}$ 53.35, $\mathrm{H}$ 3.52; found: $\mathrm{C}$ 53.45, H 3.21 .

5-(2-Bromophenyl)-1-pivalyltetrazole (1b): To a stirred suspension of $\mathrm{NaH}(60 \%$ in oil, $0.80 \mathrm{~g}, 20 \mathrm{mmol})$ in DMF $(20 \mathrm{~mL})$ was added in small portions 5-(2-bromophenyl)tetrazole $(2.25 \mathrm{~g}, 10 \mathrm{mmol})$ at $0{ }^{\circ} \mathrm{C}$, and the mixture was stirred at $\mathrm{RT}$ for about 1 hour until $\mathrm{NaH}$ disappearance. To this sodio-tetrazole mixture, pivaloyl chloride (1.45 g, $12 \mathrm{mmol})$ was added and the resulting mixture was stirred at RT for $12 \mathrm{~h}$. Then, the reaction mixture was quenched with saturated $\mathrm{NH}_{4} \mathrm{Cl}$ solution $(25 \mathrm{~mL})$, diluted with water $(25 \mathrm{~mL})$, and extracted with AcOEt $(3 \times 50 \mathrm{~mL})$. The organic phase was washed with brine $(50 \mathrm{~mL})$, dried $\left(\mathrm{Na}_{2} \mathrm{SO}_{4}\right)$ and filtered. After removal of all volatiles, the residue was purified by flash chromatography on silica gel (pentane-Et $\left.{ }_{2} \mathrm{O}, 85: 15\right)$ to afford $\mathbf{1 b}(2.41 \mathrm{~g}, 78 \%)$ as a pale yellow oil. ${ }^{1} \mathrm{H}$ NMR $\left(400 \mathrm{MHz}, \mathrm{CDCl}_{3}\right) \delta(\mathrm{ppm}) 7.96(\mathrm{dd}, J=1.8$ and $7.7 \mathrm{~Hz}, 1 \mathrm{H}), 7.76(\mathrm{dd}, J=1.3$ and $8.0 \mathrm{~Hz}$, $1 \mathrm{H}), 7.47(\mathrm{dt}, J=1.3$ and $7.5 \mathrm{~Hz}, 1 \mathrm{H}), 7.40(\mathrm{dd}, J=1.8$ and $7.9 \mathrm{~Hz}, 1 \mathrm{H}), 1.52(\mathrm{~s}, 9 \mathrm{H}) .{ }^{13} \mathrm{C}$ NMR $\left(100 \mathrm{MHz}, \mathrm{CDCl}_{3}\right) \delta(\mathrm{ppm}) 173.8,163.6,134.4,132.3,131.7,127.5,125.8,121.6$, 32.5, 28.2. Elemental analysis: calcd (\%) for $\mathrm{C}_{12} \mathrm{H}_{13} \mathrm{BrN}_{4} \mathrm{O}$ (309.17): C 46.62, H 4.24; found: C 46.89, H 4.31 .

5-(2-Bromophenyl)-1-methyltetrazole (1c): To a stirred suspension of $\mathrm{NaH}(60 \%$ in oil, $0.80 \mathrm{~g}, 20 \mathrm{mmol})$ in DMF $(20 \mathrm{~mL})$ was added in small portions 5-(2-bromophenyl)tetrazole 
(2.25 g, $10 \mathrm{mmol}$ ) at $0{ }^{\circ} \mathrm{C}$, and the mixture was stirred at RT for about 1 hour until $\mathrm{NaH}$ disappearance. To this sodio-tetrazole mixture, methyl iodide $(1.70 \mathrm{~g}, 12 \mathrm{mmol})$ was added and the resulting mixture was stirred at RT for $12 \mathrm{~h}$. Then, the reaction mixture was quenched with saturated $\mathrm{NH}_{4} \mathrm{Cl}$ solution $(25 \mathrm{~mL})$, diluted with water $(25 \mathrm{~mL})$, and extracted with AcOEt $(3 \times 50 \mathrm{~mL})$. The organic phase was washed with brine $(50 \mathrm{~mL})$, dried $\left(\mathrm{Na}_{2} \mathrm{SO}_{4}\right)$ and filtered. After removal of all volatiles, the residue was purified by flash chromatography on silica gel (pentane-Et $\left.{ }_{2} \mathrm{O}, 85: 15\right)$ to afford $1 \mathrm{c}(2.18 \mathrm{~g}, 91 \%)$ as a white solid $(\mathrm{mp}=43-45$ $\left.{ }^{\circ} \mathrm{C}\right) .{ }^{1} \mathrm{H}$ NMR $\left(400 \mathrm{MHz}, \mathrm{CDCl}_{3}\right) \delta(\mathrm{ppm}) 7.84(\mathrm{dd}, J=1.8$ and $7.7 \mathrm{~Hz}, 1 \mathrm{H}), 7.73(\mathrm{dd}, J=1.3$ and $8.0 \mathrm{~Hz}, 1 \mathrm{H}), 7.42(\mathrm{dt}, J=1.3$ and $7.6 \mathrm{~Hz}, 1 \mathrm{H}), 7.32(\mathrm{dd}, J=1.8$ and $7.6 \mathrm{~Hz}, 1 \mathrm{H}), 4.43(\mathrm{~s}$, $3 \mathrm{H}) .{ }^{13} \mathrm{C}$ NMR $\left(100 \mathrm{MHz}, \mathrm{CDCl}_{3}\right) \delta$ (ppm) 164.3, 134.1, 131.7, 131.2, 128.5, 127.5, 122.0, 39.7. Elemental analysis: calcd (\%) for $\mathrm{C}_{8} \mathrm{H}_{7} \mathrm{BrN}_{4}$ (239.08): C 40.19, $\mathrm{H}$ 2.95; found: $\mathrm{C} 40.28$, H 3.06 .

General Procedure. As a typical experiment, the reaction of the $\mathrm{N}$-protected 5-(2bromophenyl)tetrazole $(0.5 \mathrm{mmol})$, (hetero)arene $(0.75 \mathrm{mmol})$, and PivOK (140 $\mathrm{mg}, 1 \mathrm{mmol})$ at $150{ }^{\circ} \mathrm{C}$ over $16 \mathrm{~h}$ in $2 \mathrm{~mL}$ of DMA in the presence of of $\mathrm{PdCl}\left(\mathrm{C}_{3} \mathrm{H}_{5}\right)(\mathrm{dppb})(6.1 \mathrm{mg}, 0.01$ mmol), under argon affords the coupling product after evaporation of the solvent and purification on silica gel.

5-(2-(1-Benzyltetrazol-5-yl)phenyl)-2-ethyl-4-methylthiazole (2): Following the general procedure using 1-benzyl-5-(2-bromophenyl)tetrazole $1 \mathrm{a}(158 \mathrm{mg}, 0.5 \mathrm{mmol})$ and 2-ethyl-4methylthiazole ( $95 \mathrm{mg}, 0.75 \mathrm{mmol}$ ), the residue was purified by flash chromatography on silica gel (pentane-Et $\left.{ }_{2} \mathrm{O}, 70: 30\right)$ to afford the desired compound $2(136 \mathrm{mg}, 75 \%)$ as a colorless oil. ${ }^{1} \mathrm{H}$ NMR $\left(400 \mathrm{MHz}, \mathrm{CDCl}_{3}\right) \delta(\mathrm{ppm}) 8.05-7.94(\mathrm{~m}, 1 \mathrm{H}), 7.55-7.47(\mathrm{~m}, 2 \mathrm{H})$, $7.47-7.42(\mathrm{~m}, 1 \mathrm{H}), 7.40-7.32(\mathrm{~m}, 3 \mathrm{H}), 7.27(\mathrm{dt}, J=2.4$ and $6.8 \mathrm{~Hz}, 2 \mathrm{H}), 5.67(\mathrm{~s}, 2 \mathrm{H}), 2.97$ $(\mathrm{q}, J=7.6 \mathrm{~Hz}, 2 \mathrm{H}), 1.95(\mathrm{~s}, 3 \mathrm{H}), 1.36(\mathrm{t}, J=7.6 \mathrm{~Hz}, 3 \mathrm{H}) .{ }^{13} \mathrm{C} \mathrm{NMR}\left(100 \mathrm{MHz}, \mathrm{CDCl}_{3}\right) \delta$ (ppm) 170.7, 164.8, 148.4, 133.2, 132.4, 131.5, 130.0, 129.8, 128.9, 128.9, 128.7, 128.4, 128.3, 128.1, 56.7, 26.8, 15.2, 14.2. Elemental analysis: calcd (\%) for $\mathrm{C}_{20} \mathrm{H}_{19} \mathrm{~N}_{5} \mathrm{~S}$ (361.46): C 66.46, H 5.30; found: C 66.59, H 5.11.

5-(2-(1-Benzyltetrazol-5-yl)phenyl)-2-isobutylthiazole (3): Following the general procedure using 1-benzyl-5-(2-bromophenyl)tetrazole $1 \mathrm{a}(158 \mathrm{mg}, 0.5 \mathrm{mmol})$ and 2-isobutylthiazole (106 $\mathrm{mg}, 0.75 \mathrm{mmol}$ ), the residue was purified by flash chromatography on silica gel (pentane-Et $\left.{ }_{2} \mathrm{O}, 70: 30\right)$ to afford the desired compound $3(148 \mathrm{mg}, 79 \%)$ as a pale yellow oil. ${ }^{1} \mathrm{H}$ NMR $\left(400 \mathrm{MHz}, \mathrm{CDCl}_{3}\right) \delta(\mathrm{ppm}) 7.86-7.78(\mathrm{~m}, 1 \mathrm{H}), 7.56-7.52(\mathrm{~m}, 1 \mathrm{H}), 7.49(\mathrm{td}, J=$ 1.9 and $7.0 \mathrm{~Hz}, 2 \mathrm{H}), 7.46(\mathrm{~s}, 1 \mathrm{H}), 7.39-7.30(\mathrm{~m}, 5 \mathrm{H}), 5.72(\mathrm{~s}, 2 \mathrm{H}), 2.79(\mathrm{~d}, J=7.2 \mathrm{~Hz}, 2 \mathrm{H})$, $2.05(\mathrm{~m}, 1 \mathrm{H}), 0.99(\mathrm{~d}, J=6.6 \mathrm{~Hz}, 6 \mathrm{H}) .{ }^{13} \mathrm{C} \mathrm{NMR}\left(100 \mathrm{MHz}, \mathrm{CDCl}_{3}\right) \delta(\mathrm{ppm}) 170.6,164.8$, 140.8, 135.6, 133.2, 131.4, 131.3, 130.7, 130.1, 129.0, 128.9, 128.5, 128.3, 127.1, 56.8, 42.3, 29.8, 22.3. Elemental analysis: calcd (\%) for $\mathrm{C}_{21} \mathrm{H}_{21} \mathrm{~N}_{5} \mathrm{~S}$ (375.49): C 67.17, H 5.64; found: C 67.31, H 5.56.

1-Benzyl-5-(2-(5-methylthiophen-2-yl)phenyl)tetrazole (4): Following the general procedure using 1-benzyl-5-(2-bromophenyl)tetrazole 1a (158 $\mathrm{mg}, 0.5 \mathrm{mmol})$ and 2methylthiophene $(74 \mathrm{mg}, 0.75 \mathrm{mmol}$ ), the residue was purified by flash chromatography on silica gel (pentane-Et $\left.{ }_{2} \mathrm{O}, 70: 30\right)$ to afford the desired compound $4(120 \mathrm{mg}, 72 \%)$ as a pale yellow oil. ${ }^{1} \mathrm{H}$ NMR $\left(400 \mathrm{MHz}, \mathrm{CDCl}_{3}\right) \delta(\mathrm{ppm}) 7.70(\mathrm{~d}, J=7.7 \mathrm{~Hz}, 1 \mathrm{H}), 7.57(\mathrm{dd}, J=1.5$ and $7.7 \mathrm{~Hz}, 1 \mathrm{H}), 7.50(\mathrm{dt}, J=1.5$ and $7.5 \mathrm{~Hz}, 1 \mathrm{H}), 7.43(\mathrm{t}, J=7.5 \mathrm{~Hz}, 1 \mathrm{H}), 7.40-7.31(\mathrm{~m}$, $5 \mathrm{H}), 6.59(\mathrm{~d}, J=3.5 \mathrm{~Hz}, 1 \mathrm{H}), 6.55(\mathrm{~d}, J=3.5 \mathrm{~Hz}, 1 \mathrm{H}), 5.77(\mathrm{~s}, 2 \mathrm{H}), 2.42(\mathrm{~s}, 3 \mathrm{H}) .{ }^{13} \mathrm{C} \mathrm{NMR}$ $\left(100 \mathrm{MHz}, \mathrm{CDCl}_{3}\right) \delta(\mathrm{ppm}) 165.4,140.4,139.3,135.1,133.5,130.8,130.0,128.9,128.8$, $128.8,128.3,127.5,126.8,126.5,125.3,56.7,15.3$. Elemental analysis: calcd (\%) for $\mathrm{C}_{19} \mathrm{H}_{16} \mathrm{~N}_{4} \mathrm{~S}$ (332.43): C 68.65, H 4.85; found: C 68.98, H 5.10. 
1-(5-(2-(1-Benzyltetrazol-5-yl)phenyl)-4-methylthiophen-2-yl)ethan-1-one (5): Following the general procedure using 1-benzyl-5-(2-bromophenyl)tetrazole $1 \mathrm{a}(158 \mathrm{mg}, 0.5 \mathrm{mmol})$ and 1-(4-methylthiophen-2-yl)ethan-1-one (105 $\mathrm{mg}, 0.75 \mathrm{mmol})$, the residue was purified by flash chromatography on silica gel (pentane-Et $2 \mathrm{O}, 60: 40)$ to afford the desired compound 5 (139 $\mathrm{mg}, 74 \%)$ as a pale yellow oil. ${ }^{1} \mathrm{H}$ NMR $\left(400 \mathrm{MHz}, \mathrm{CDCl}_{3}\right) \delta(\mathrm{ppm}) 8.08-8.01(\mathrm{~m}, 1 \mathrm{H})$, $7.60-7.49(\mathrm{~m}, 2 \mathrm{H}), 7.46-7.40(\mathrm{~m}, 1 \mathrm{H}), 7.37-7.33(\mathrm{~m}, 3 \mathrm{H}), 7.32(\mathrm{~s}, 1 \mathrm{H}), 7.27-7.21(\mathrm{~m}$, 2H), $5.66(\mathrm{~s}, 2 \mathrm{H}), 2.52(\mathrm{~s}, 3 \mathrm{H}), 1.71(\mathrm{~s}, 3 \mathrm{H}) .{ }^{13} \mathrm{C} \mathrm{NMR}\left(100 \mathrm{MHz}, \mathrm{CDCl}_{3}\right) \delta(\mathrm{ppm}) 190.6$, $164.8,145.5,141.9,136.4,134.8,133.3,132.8,132.0,130.0,130.0,129.2,129.0,128.5$, 127.8, 56.8, 26.8, 14.1. Elemental analysis: calcd (\%) for $\mathrm{C}_{21} \mathrm{H}_{18} \mathrm{~N}_{4} \mathrm{OS}(374.46): \mathrm{C} 67.36, \mathrm{H}$ 4.85, found: C 67.49, H 5.19.

5-(2-(Benzo $[b]$ thiophen-2-yl)phenyl)-1-benzyltetrazole (6): Following the general procedure using 1-benzyl-5-(2-bromophenyl)tetrazole 1a $(158 \mathrm{mg}, 0.5 \mathrm{mmol})$ and benzo[b]thiophene $(101 \mathrm{mg}, 0.75 \mathrm{mmol})$, the residue was purified by flash chromatography on silica gel (pentane-Et $\left.{ }_{2} \mathrm{O}, 80: 20\right)$ to afford the desired compound $6(127 \mathrm{mg}, 69 \%)$ as a yellow solid $\left(\mathrm{mp}=76-78^{\circ} \mathrm{C}\right) .{ }^{1} \mathrm{H}$ NMR $\left(400 \mathrm{MHz}, \mathrm{CDCl}_{3}\right) \delta(\mathrm{ppm}) 7.85(\mathrm{dd}, J=1.2$ and 7.1 $\mathrm{Hz}, 1 \mathrm{H}), 7.76(\mathrm{ddd}, J=0.8,1.8$ and $7.0 \mathrm{~Hz}, 1 \mathrm{H}), 7.70-7.63(\mathrm{~m}, 2 \mathrm{H}), 7.60-7.47(\mathrm{~m}, 2 \mathrm{H})$, $7.42-7.31(\mathrm{~m}, 2 \mathrm{H}), 7.30-7.23(\mathrm{~m}, 1 \mathrm{H}), 7.20-7.15(\mathrm{~m}, 4 \mathrm{H}), 7.08(\mathrm{~s}, 1 \mathrm{H}), 5.68(\mathrm{~s}, 2 \mathrm{H}) .{ }^{13} \mathrm{C}$ NMR $\left(100 \mathrm{MHz}, \mathrm{CDCl}_{3}\right) \delta(\mathrm{ppm}) 165.0,142.2,140.5,140.0,134.7,133.1,131.4,130.7$, 130.1, 128.8, 128.7, 128.5, 128.2, 127.1, 124.2, 124.1, 123.7, 123.6, 122.1, 56.7. Elemental analysis: calcd (\%) for $\mathrm{C}_{22} \mathrm{H}_{16} \mathrm{~N}_{4} \mathrm{~S}$ (368.45): C 71.71, H 4.38, found: C 72.00, H 4.39.

1-Benzyl-5-(2-(5-butylfuran-2-yl)phenyl)tetrazole (7): Following the general procedure using 1-benzyl-5-(2-bromophenyl)tetrazole 1a (158 $\mathrm{mg}, 0.5 \mathrm{mmol})$ and 2-n-butylfuran (93 $\mathrm{mg}, 0.75 \mathrm{mmol}$ ), the residue was purified by flash chromatography on silica gel (pentane$\left.\mathrm{Et}_{2} \mathrm{O}, 80: 20\right)$ to afford the desired compound $7(147 \mathrm{mg}, 82 \%)$ as a yellow oil. ${ }^{1} \mathrm{H}$ NMR (400 $\left.\mathrm{MHz}, \mathrm{CDCl}_{3}\right) \delta(\mathrm{ppm}) 7.73(\mathrm{dd}, J=1.3$ and $7.9 \mathrm{~Hz}, 1 \mathrm{H}), 7.61(\mathrm{dd}, J=1.4$ and $7.7 \mathrm{~Hz}, 1 \mathrm{H})$, $7.51(\mathrm{dt}, J=1.4$ and $7.7 \mathrm{~Hz}, 1 \mathrm{H}), 7.47-7.37(\mathrm{~m}, 4 \mathrm{H}), 7.36(\mathrm{dd}, J=1.3$ and $7.6 \mathrm{~Hz}, 1 \mathrm{H}), 6.01$ $(\mathrm{d}, J=3.2 \mathrm{~Hz}, 1 \mathrm{H}), 5.90(\mathrm{~d}, J=3.2 \mathrm{~Hz}, 1 \mathrm{H}), 5.82(\mathrm{~s}, 2 \mathrm{H}), 2.43(\mathrm{t}, J=7.6 \mathrm{~Hz}, 2 \mathrm{H}), 1.55-1.40$ $(\mathrm{m}, 2 \mathrm{H}), 1.39-1.21(\mathrm{~m}, 2 \mathrm{H}), 0.92(\mathrm{t}, J=7.3 \mathrm{~Hz}, 3 \mathrm{H}) .{ }^{13} \mathrm{C} \mathrm{NMR}\left(100 \mathrm{MHz}, \mathrm{CDCl}_{3}\right) \delta(\mathrm{ppm})$ $165.8,156.6,150.2,133.4,131.3,131.1,130.1,129.0,128.9,128.5,127.7,127.0,124.4$, $109.4,106.5,56.7,30.0,27.6,22.2,13.8$. Elemental analysis: calcd (\%) for $\mathrm{C}_{22} \mathrm{H}_{22} \mathrm{~N}_{4} \mathrm{O}$ (358.44): C 73.72, H 6.19, found: C 74.02, H 6.37.

1-(5-(2-(1-Benzyltetrazol-5-yl)phenyl)furan-2-yl)butan-1-one (8): Following the general procedure using 1-benzyl-5-(2-bromophenyl)tetrazole $1 \mathrm{a}(158 \mathrm{mg}, 0.5 \mathrm{mmol})$ and 1-(furan-2yl)butan-1-one (104 $\mathrm{mg}, 0.75 \mathrm{mmol})$, the residue was purified by flash chromatography on silica gel (pentane-Et $\left.{ }_{2} \mathrm{O}, 70: 30\right)$ to afford the desired compound $\mathbf{8}(164 \mathrm{mg}, 88 \%)$ as a brown yellow oil. ${ }^{1} \mathrm{H}$ NMR $\left(400 \mathrm{MHz}, \mathrm{CDCl}_{3}\right) \delta(\mathrm{ppm}) 7.86-7.70(\mathrm{~m}, 2 \mathrm{H}), 7.58(\mathrm{dt}, J=1.5$ and $7.6 \mathrm{~Hz}, 1 \mathrm{H}), 7.52(\mathrm{dt}, J=1.4$ and $7.6 \mathrm{~Hz}, 1 \mathrm{H}), 7.42-7.35(\mathrm{~m}, 5 \mathrm{H}), 7.09(\mathrm{~d}, J=3.6 \mathrm{~Hz}, 1 \mathrm{H})$, $6.28(\mathrm{~d}, J=3.7 \mathrm{~Hz}, 1 \mathrm{H}), 5.78(\mathrm{~s}, 2 \mathrm{H}), 2.50(\mathrm{t}, J=7.3 \mathrm{~Hz}, 2 \mathrm{H}), 1.63$ (sext., $J=7.4 \mathrm{~Hz}, 2 \mathrm{H})$, $0.93(\mathrm{t}, J=7.4 \mathrm{~Hz}, 3 \mathrm{H}) .{ }^{13} \mathrm{C}$ NMR $\left(100 \mathrm{MHz}, \mathrm{CDCl}_{3}\right) \delta(\mathrm{ppm}) 189.5,165.0,155.6,152.3$, $133.1,131.1,130.3,129.6,129.2,129.2,129.1,129.1,128.5,125.9,117.8,111.0,56.9,40.1$, 17.6, 13.8. Elemental analysis: calcd (\%) for $\mathrm{C}_{22} \mathrm{H}_{20} \mathrm{~N}_{4} \mathrm{O}_{2}$ (372.42): C 70.95, H 5.41, found: $\mathrm{C}$ $71.28, \mathrm{H} 5.17$.

1-Benzyl-5-(2-(1-methylpyrrol-2-yl)phenyl)tetrazole (9): Following the general procedure using 1-benzyl-5-(2-bromophenyl)tetrazole $1 \mathrm{a}(158 \mathrm{mg}, 0.5 \mathrm{mmol})$ and $N$-methylpyrrole (162 $\mathrm{mg}, 2 \mathrm{mmol}$ ), the residue was purified by flash chromatography on silica gel (pentane-Et ${ }_{2} \mathrm{O}$, 70:30) to afford the desired compound $9(145 \mathrm{mg}, 92 \%)$ as a brown oil. ${ }^{1} \mathrm{H}$ NMR $(400 \mathrm{MHz}$, $\left.\mathrm{CDCl}_{3}\right) \delta(\mathrm{ppm}) 8.12-7.97(\mathrm{~m}, 1 \mathrm{H}), 7.58-7.45(\mathrm{~m}, 3 \mathrm{H}), 7.42-7.36(\mathrm{~m}, 3 \mathrm{H}), 7.36-7.28$ 
$(\mathrm{m}, 2 \mathrm{H}), 6.60-6.50(\mathrm{~m}, 1 \mathrm{H}), 6.22-6.13(\mathrm{~m}, 1 \mathrm{H}), 6.04(\mathrm{dd}, J=1.8$ and $3.5 \mathrm{~Hz}, 1 \mathrm{H}), 5.69(\mathrm{~s}$, $2 \mathrm{H}), 3.06(\mathrm{~s}, 3 \mathrm{H}) .{ }^{13} \mathrm{C} \mathrm{NMR}\left(100 \mathrm{MHz}, \mathrm{CDCl}_{3}\right) \delta(\mathrm{ppm}) 165.3,133.4,132.9,132.5,132.5$, 129.8, 129.6, 128.9, 128.8, 128.6, 128.2, 128.1, 122.1, 108.7, 107.4, 56.6, 33.9. Elemental analysis: calcd (\%) for $\mathrm{C}_{19} \mathrm{H}_{17} \mathrm{~N}_{5}$ (315.37): C 72.36, H 5.43, found: C 72.36, H 5.22.

3-(2-(1-Benzyltetrazol-5-yl)phenyl)imidazo[1,2-a]pyridine (10): Following the general procedure using 1-benzyl-5-(2-bromophenyl)tetrazole $1 \mathrm{a}(158 \mathrm{mg}, 0.5 \mathrm{mmol})$ and imidazo[1,2-a]pyridine (89 $\mathrm{mg}, 0.75 \mathrm{mmol}$ ), the residue was purified by flash chromatography on silica gel (pentane-Et ${ }_{2} \mathrm{O}, 70: 30$ ) to afford the desired compound 10 (173 $\mathrm{mg}, 98 \%)$ as a yellow solid $\left(\mathrm{mp}=170-172{ }^{\circ} \mathrm{C}\right) .{ }^{1} \mathrm{H}$ NMR $\left(400 \mathrm{MHz}, \mathrm{CDCl}_{3}\right) \delta(\mathrm{ppm}) 8.35-$ $8.21(\mathrm{~m}, 1 \mathrm{H}), 7.67-7.58(\mathrm{~m}, 4 \mathrm{H}), 7.59-7.53(\mathrm{~m}, 1 \mathrm{H}), 7.43(\mathrm{~d}, J=7.0 \mathrm{~Hz}, 1 \mathrm{H}), 7.35-7.23$ $(\mathrm{m}, 3 \mathrm{H}), 7.07(\mathrm{ddd}, J=1.3,6.7$ and $9.2 \mathrm{~Hz}, 1 \mathrm{H}), 7.05-6.98(\mathrm{~m}, 2 \mathrm{H}), 6.45(\mathrm{dd}, J=6.7$ and $6.8 \mathrm{~Hz}, 1 \mathrm{H}), 5.42(\mathrm{~s}, 2 \mathrm{H}) .{ }^{13} \mathrm{C}$ NMR $\left(100 \mathrm{MHz}, \mathrm{CDCl}_{3}\right) \delta(\mathrm{ppm}) 164.2,145.4,132.9,132.9$, $132.7,130.6,130.0,129.5,128.9,128.8,128.4,127.7,127.7,124.4,123.9,123.6,117.7$, 111.8, 56.6. Elemental analysis: calcd (\%) for $\mathrm{C}_{21} \mathrm{H}_{16} \mathrm{~N}_{6}(352.39)$ : $\mathrm{C} 71.58, \mathrm{H} \mathrm{4.58}$, found: $\mathrm{C}$ $71.69, \mathrm{H} 4.37$.

1-Benzyl-5-(2',3',4',5',6'-pentafluoro-[1,1'-biphenyl]-2-yl)tetrazole (11): Following the general procedure using 1-benzyl-5-(2-bromophenyl)tetrazole $1 \mathrm{a}(158 \mathrm{mg}, 0.5 \mathrm{mmol})$ and pentafluorobenzene $(126 \mathrm{mg}, 0.75 \mathrm{mmol})$, the residue was purified by flash chromatography on silica gel (pentane-Et $\left.{ }_{2} \mathrm{O}, 70: 30\right)$ to afford the desired compound $11(151 \mathrm{mg}, 75 \%)$ as a yellow solid $\left(\mathrm{mp}=99-102{ }^{\circ} \mathrm{C}\right) .{ }^{1} \mathrm{H} \operatorname{NMR}\left(400 \mathrm{MHz}, \mathrm{CDCl}_{3}\right) \delta(\mathrm{ppm}) 8.29$ (dd, $J=1.6$ and $7.9 \mathrm{~Hz}, 1 \mathrm{H}), 7.64(\mathrm{dt}, J=1.6,7.6 \mathrm{~Hz}, 1 \mathrm{H}), 7.59(\mathrm{dt}, J=1.6,7.5 \mathrm{~Hz}, 1 \mathrm{H}), 7.47-7.34(\mathrm{~m}, 4 \mathrm{H})$, $7.28-7.21(\mathrm{~m}, 2 \mathrm{H}), 5.67(\mathrm{~s}, 2 \mathrm{H}) .{ }^{13} \mathrm{C}$ NMR $\left(100 \mathrm{MHz}, \mathrm{CDCl}_{3}\right) \delta(\mathrm{ppm}) 164.2,144.3(\mathrm{dm}, J$ $=253.1 \mathrm{~Hz}), 140.7(\mathrm{dm}, J=253.1 \mathrm{~Hz}), 137.4(\mathrm{dm}, J=253.1 \mathrm{~Hz}), 132.8,131.8,130.2,130.0$, 129.7, 129.1, 128.9, 128.4, 127.5, 125.1, $115.4(\mathrm{t}, J=8.5 \mathrm{~Hz}), 56.8$. Elemental analysis: calcd (\%) for $\mathrm{C}_{20} \mathrm{H}_{11} \mathrm{~F}_{5} \mathrm{~N}_{4}$ (402.32): C 59.71, H 2.76, found: C 59.98, H 2.51.

2'-(1-Benzyltetrazol-5-yl)-2,6-difluoro-[1,1'-biphenyl]-4-carbonitrile (12): Following the general procedure using 1-benzyl-5-(2-bromophenyl)tetrazole 1a (158 $\mathrm{mg}, 0.5 \mathrm{mmol})$ and 3,5-difluorobenzonitrile (104 $\mathrm{mg}, 0.75 \mathrm{mmol})$, the residue was purified by flash chromatography on silica gel (pentane-Et $\mathrm{H}_{2} \mathrm{O}, 80: 20$ ) to afford the desired compound 12 (125 $\mathrm{mg}, 67 \%)$ as a yellow solid $\left(\mathrm{mp}=172-174{ }^{\circ} \mathrm{C}\right) .{ }^{1} \mathrm{H} \mathrm{NMR}\left(400 \mathrm{MHz}, \mathrm{CDCl}_{3}\right) \delta(\mathrm{ppm}) 8.29$ (dd, $J=1.7$ and $7.5 \mathrm{~Hz}, 1 \mathrm{H}), 7.70-7.53(\mathrm{~m}, 2 \mathrm{H}), 7.47-7.33(\mathrm{~m}, 4 \mathrm{H}), 7.26-7.15(\mathrm{~m}, 2 \mathrm{H})$, $7.08-6.96(\mathrm{~m}, 2 \mathrm{H}), 5.63(\mathrm{~s}, 2 \mathrm{H}) .{ }^{13} \mathrm{C}$ NMR $\left(100 \mathrm{MHz}, \mathrm{CDCl}_{3}\right) \delta(\mathrm{ppm}) 164.4,160.0,(\mathrm{dd}, J$ $=7.7$ and $252.4 \mathrm{~Hz}), 133.0,131.4,130.1,129.9,129.6,129.2,129.0,128.4,127.1,126.2$, $123.8(\mathrm{t}, J=20.4 \mathrm{~Hz}), 116.8(\mathrm{t}, J=3.5 \mathrm{~Hz}), 115.2(\mathrm{~d}, J=31.8 \mathrm{~Hz}), 112.6(\mathrm{t}, J=12.8 \mathrm{~Hz})$, 56.8. Elemental analysis: calcd (\%) for $\mathrm{C}_{21} \mathrm{H}_{13} \mathrm{~F}_{2} \mathrm{~N}_{5}$ (373.36): $\mathrm{C}$ 67.56, $\mathrm{H}$ 3.51; found: $\mathrm{C}$ $67.63, \mathrm{H} 3.19$.

5-(2-(1-Pivalyltetrazol-5-yl)phenyl)-2-ethyl-4-methylthiazole (13): Following the general procedure using 5-(2-bromophenyl)-1-pivalyltetrazole $\mathbf{1 b}(155 \mathrm{mg}, 0.5 \mathrm{mmol})$ and 2-ethyl-4methylthiazole $(95 \mathrm{mg}, 0.75 \mathrm{mmol})$, the residue was purified by flash chromatography on silica gel (pentane-Et $\left.{ }_{2} \mathrm{O}, 70: 30\right)$ to afford the desired compound $\mathbf{1 3}$ (156 $\mathrm{mg}, 88 \%$ ) as a pale yellow oil. ${ }^{1} \mathrm{H}$ NMR $\left(400 \mathrm{MHz}, \mathrm{CDCl}_{3}\right) \delta(\mathrm{ppm}) 8.14-8.07(\mathrm{~m}, 1 \mathrm{H}), 7.59-7.47(\mathrm{~m}, 2 \mathrm{H})$, $7.43(\mathrm{dd}, J=2.0,6.4 \mathrm{~Hz}, 1 \mathrm{H}), 2.98(\mathrm{q}, J=7.6 \mathrm{~Hz}, 2 \mathrm{H}), 2.08(\mathrm{~s}, 3 \mathrm{H}), 1.37(\mathrm{t}, J=7.6 \mathrm{~Hz}, 3 \mathrm{H})$, $1.25(\mathrm{~s}, 9 \mathrm{H}) .{ }^{13} \mathrm{C}$ NMR $\left(100 \mathrm{MHz}, \mathrm{CDCl}_{3}\right) \delta(\mathrm{ppm}) 173.5,171.1,164.4,148.9,132.5,131.3$, 131.0, 130.0, 128.9, 127.8, 125.1, 32.3, 27.9, 26.8, 15.1, 14.3. Elemental analysis: calcd (\%) for $\mathrm{C}_{18} \mathrm{H}_{21} \mathrm{~N}_{5} \mathrm{OS}$ (355.46): C 60.82, H 5.95; found: C 61.08, H 6.11.

5-(2-(1-Pivalyltetrazol-5-yl)phenyl)-2-isobutylthiazole (14): Following the general procedure using 5-(2-bromophenyl)-1-pivalyltetrazole $\mathbf{1 b}(155 \mathrm{mg}, 0.5 \mathrm{mmol})$ and 2- 
isobutylthiazole (106 $\mathrm{mg}, 0.75 \mathrm{mmol}$ ), the residue was purified by flash chromatography on silica gel (pentane- $\left.\mathrm{Et}_{2} \mathrm{O}, 70: 30\right)$ to afford the desired compound 14 (168 $\left.\mathrm{mg}, 91 \%\right)$ as a pale yellow oil. ${ }^{1} \mathrm{H}$ NMR $\left(400 \mathrm{MHz}, \mathrm{CDCl}_{3}\right) \delta(\mathrm{ppm}) 7.98(\mathrm{dd}, J=1.6$ and $7.8 \mathrm{~Hz}, 1 \mathrm{H}), 7.61-$ $7.49(\mathrm{~m}, 3 \mathrm{H}), 7.47(\mathrm{~s}, 1 \mathrm{H}), 2.86(\mathrm{~d}, J=7.2 \mathrm{~Hz}, 2 \mathrm{H}), 2.17-2.06(\mathrm{~m}, 1 \mathrm{H}), 1.30(\mathrm{~s}, 9 \mathrm{H}), 1.03$ $(\mathrm{d}, J=6.7 \mathrm{~Hz}, 6 \mathrm{H}) .{ }^{13} \mathrm{C}$ NMR $\left(100 \mathrm{MHz}, \mathrm{CDCl}_{3}\right) \delta(\mathrm{ppm}) 173.7,171.0,164.3,140.7,135.0$, $131.7,131.2,131.1,130.6,128.8,124.2,42.4,32.3,29.7,28.0,22.3$. Elemental analysis: calcd (\%) for $\mathrm{C}_{19} \mathrm{H}_{23} \mathrm{~N}_{5} \mathrm{OS}$ (369.48): C 61.76, H 6.27; found: $\mathrm{C}$ 62.07, H 6.35.

5-(2-(5-Methylthiophen-2-yl)phenyl)-1-pivalyltetrazole (15): Following the general procedure using 5-(2-bromophenyl)-1-pivalyltetrazole $\mathbf{1 b}(155 \mathrm{mg}, 0.5 \mathrm{mmol})$ and 2methylthiophene (74 $\mathrm{mg}, 0.75 \mathrm{mmol}$ ), the residue was purified by flash chromatography on silica gel (pentane-Et $\left.{ }_{2} \mathrm{O}, 70: 30\right)$ to afford the desired compound $\mathbf{1 5}(139 \mathrm{mg}, 85 \%$ ) as a yellow solid $\left(\mathrm{mp}=88-90{ }^{\circ} \mathrm{C}\right) .{ }^{1} \mathrm{H}$ NMR $\left(400 \mathrm{MHz}, \mathrm{CDCl}_{3}\right) \delta(\mathrm{ppm}) 7.92-7.86(\mathrm{~m}, 1 \mathrm{H}), 7.57-7.50$ $(\mathrm{m}, 2 \mathrm{H}), 7.44(\mathrm{ddd}, J=3.8,5.1$ and $7.7 \mathrm{~Hz}, 1 \mathrm{H}), 7.01-6.24(\mathrm{~m}, 2 \mathrm{H}), 2.47(\mathrm{~s}, 3 \mathrm{H}), 1.29(\mathrm{~s}$, 9H). ${ }^{13} \mathrm{C}$ NMR $\left(100 \mathrm{MHz}, \mathrm{CDCl}_{3}\right) \delta$ (ppm) 173.6, 164.9, 140.8, 138.9, 135.0, 131.1, 131.0, 130.6, 127.9, 126.6, 125.4, 123.8, 32.2, 27.9, 15.2. Elemental analysis: calcd (\%) for $\mathrm{C}_{17} \mathrm{H}_{18} \mathrm{~N}_{4} \mathrm{OS}$ (326.42): C 62.55, H 5.56; found: C 62.75, H 5.69.

\section{1-(5-(2-(1-Pivalyltetrazol-5-yl)phenyl)-4-methylthiophen-2-yl)ethan-1-one}

(16): Following the general procedure using 5-(2-bromophenyl)-1-pivalyltetrazole 1b (155 mg, 0.5 $\mathrm{mmol})$ and 1-(4-methylthiophen-2-yl)ethan-1-one (105 $\mathrm{mg}, 0.75 \mathrm{mmol})$, the residue was purified by flash chromatography on silica gel (pentane-Et ${ }_{2} \mathrm{O}, 60: 40$ ) to afford the desired compound $16(153 \mathrm{mg}, 83 \%)$ as a pale yellow oil. ${ }^{1} \mathrm{H}$ NMR $\left(400 \mathrm{MHz}, \mathrm{CDCl}_{3}\right) \delta(\mathrm{ppm}) 8.17$ $-8.11(\mathrm{~m}, 1 \mathrm{H}), 7.64-7.54(\mathrm{~m}, 2 \mathrm{H}), 7.49(\mathrm{~s}, 1 \mathrm{H}), 7.47-7.40(\mathrm{~m}, 1 \mathrm{H}), 2.54(\mathrm{~s}, 3 \mathrm{H}), 1.92(\mathrm{~s}$, $3 \mathrm{H}), 1.24(\mathrm{~s}, 9 \mathrm{H}) .{ }^{13} \mathrm{C} \mathrm{NMR}\left(100 \mathrm{MHz}, \mathrm{CDCl}_{3}\right) \delta(\mathrm{ppm}) 190.2,173.3,164.0,144.4,142.3$, $136.5,134.2,132.3,131.8,130.9,129.7,129.1,124.4,32.0,27.6,16.5,13.7$. Elemental analysis: calcd (\%) for $\mathrm{C}_{19} \mathrm{H}_{20} \mathrm{~N}_{4} \mathrm{O}_{2} \mathrm{~S}$ (368.45): C 61.94, H 5.47, found: C 62.09, H 5.11.

5-(2-(Benzo $[b]$ thiophen-2-yl)phenyl)-1-pivalyltetrazole (17): Following the general procedure using 5-(2-bromophenyl)-1-pivalyltetrazole 1 b $(155 \mathrm{mg}, 0.5 \mathrm{mmol})$ and benzo[b]thiophene $(101 \mathrm{mg}, 0.75 \mathrm{mmol})$, the residue was purified by flash chromatography on silica gel (pentane-Et $\left.{ }_{2} \mathrm{O}, 80: 20\right)$ to afford the desired compound $17(143 \mathrm{mg}, 79 \%$ ) as a pale yellow oil. ${ }^{1} \mathrm{H} \mathrm{NMR}\left(400 \mathrm{MHz}, \mathrm{CDCl}_{3}\right) \delta(\mathrm{ppm}) 8.01$ (dd, $J=1.6$ and $\left.7.6 \mathrm{~Hz}, 1 \mathrm{H}\right), 7.82$ $(\mathrm{dd}, J=2.0$ and $7.5 \mathrm{~Hz}, 1 \mathrm{H}), 7.72(\mathrm{dd}, J=2.0$ and $7.8 \mathrm{~Hz}, 1 \mathrm{H}), 7.67(\mathrm{dd}, J=1.5$ and $7.7 \mathrm{~Hz}$, $1 \mathrm{H}), 7.62(\mathrm{dt}, J=1.6$ and $7.5 \mathrm{~Hz}, 1 \mathrm{H}), 7.55(\mathrm{dt}, J=1.5$ and $7.5 \mathrm{~Hz}, 1 \mathrm{H}), 7.41-7.30(\mathrm{~m}, 2 \mathrm{H})$, $7.10(\mathrm{~s}, 1 \mathrm{H}), 1.10(\mathrm{~s}, 9 \mathrm{H}) .{ }^{13} \mathrm{C} \mathrm{NMR}\left(100 \mathrm{MHz}, \mathrm{CDCl}_{3}\right) \delta(\mathrm{ppm}) 173.7,164.6,141.5,140.3$, 139.9, 134.5, 131.3, 131.2, 130.6, 128.8, 124.5, 124.5, 124.3, 123.6, 123.5, 122.0, 32.2, 27.7. Elemental analysis: calcd (\%) for $\mathrm{C}_{20} \mathrm{H}_{18} \mathrm{~N}_{4} \mathrm{OS}$ (362.45): C 66.28, H 5.01, found: C 66.49, H 5.34 .

5-(2-(5-Butylfuran-2-yl)phenyl)-1-pivalyltetrazole (18): Following the general procedure using 5-(2-bromophenyl)-1-pivalyltetrazole $\mathbf{1 b}$ (155 $\mathrm{mg}, 0.5 \mathrm{mmol})$ and 2- $n$-butylfuran (93 $\mathrm{mg}, 0.75 \mathrm{mmol}$ ), the residue was purified by flash chromatography on silica gel (pentane$\left.\mathrm{Et}_{2} \mathrm{O}, 80: 20\right)$ to afford the desired compound $\mathbf{1 8}(167 \mathrm{mg}, 95 \%)$ as a brown oil. ${ }^{1} \mathrm{H}$ NMR (400 $\left.\mathrm{MHz}, \mathrm{CDCl}_{3}\right) \delta(\mathrm{ppm}) 7.76(\mathrm{dd}, J=0.9$ and $8.0 \mathrm{~Hz}, 1 \mathrm{H}), 7.71(\mathrm{dd}, J=1.1$ and $7.7 \mathrm{~Hz}, 1 \mathrm{H})$, $7.56(\mathrm{dt}, J=1.4$ and $7.7 \mathrm{~Hz}, 1 \mathrm{H}), 7.39(\mathrm{dt}, J=1.3$ and $7.6 \mathrm{~Hz}, 1 \mathrm{H}), 6.19(\mathrm{~d}, J=3.3 \mathrm{~Hz}, 1 \mathrm{H})$, $6.02(\mathrm{~d}, J=3.3 \mathrm{~Hz}, 1 \mathrm{H}), 2.58(\mathrm{t}, J=7.5 \mathrm{~Hz}, 2 \mathrm{H}), 1.66-1.53(\mathrm{~m}, 2 \mathrm{H}), 1.40(\mathrm{~s}, 9 \mathrm{H}), 1.40-$ $1.31(\mathrm{~m}, 2 \mathrm{H}), 0.93(\mathrm{t}, J=7.5 \mathrm{~Hz}, 3 \mathrm{H}) .{ }^{13} \mathrm{C} \mathrm{NMR}\left(100 \mathrm{MHz}, \mathrm{CDCl}_{3}\right) \delta(\mathrm{ppm}) 173.5,165.0$, $157.2,149.8,131.3,131.2,131.1,127.8,127.3,121.3,109.5,106.8,32.4,29.9,28.2,27.8$, 22.3, 13.8. Elemental analysis: calcd (\%) for $\mathrm{C}_{20} \mathrm{H}_{24} \mathrm{~N}_{4} \mathrm{O}_{2}$ (352.43): C 68.16, H 6.86, found: C 68.24, H 6.54 . 
1-(5-(2-(1-Pivalyltetrazol-5-yl)phenyl)furan-2-yl)butan-1-one (19): Following the general procedure using 5-(2-bromophenyl)-1-pivalyltetrazole $\mathbf{1 b}(155 \mathrm{mg}, 0.5 \mathrm{mmol})$ and 1-(furan-2yl)butan-1-one (104 $\mathrm{mg}, 0.75 \mathrm{mmol})$, the residue was purified by flash chromatography on silica gel (pentane- $\left.\mathrm{Et}_{2} \mathrm{O}, 70: 30\right)$ to afford the desired compound 19 (178 $\left.\mathrm{mg}, 97 \%\right)$ as a pale green oil. ${ }^{1} \mathrm{H}$ NMR $\left(400 \mathrm{MHz}, \mathrm{CDCl}_{3}\right) \delta(\mathrm{ppm}) 7.93(\mathrm{dd}, J=1.5$ and $7.8 \mathrm{~Hz}, 1 \mathrm{H}), 7.79(\mathrm{dd}, J$ $=1.4$ and $7.8 \mathrm{~Hz}, 1 \mathrm{H}), 7.65(\mathrm{dt}, J=1.3$ and $7.7 \mathrm{~Hz}, 1 \mathrm{H}), 7.57(\mathrm{dt}, J=1.3$ and $7.5 \mathrm{~Hz}, 1 \mathrm{H})$, $7.22(\mathrm{~d}, J=3.6 \mathrm{~Hz}, 1 \mathrm{H}), 6.49(\mathrm{~d}, J=3.6 \mathrm{~Hz}, 1 \mathrm{H}), 2.70(\mathrm{~d}, J=7.4 \mathrm{~Hz}, 2 \mathrm{H}), 1.73$ (sext., $J=7.4$ $\mathrm{Hz}, 2 \mathrm{H}), 1.36(\mathrm{~s}, 9 \mathrm{H}), 0.99(\mathrm{t}, J=7.4 \mathrm{~Hz}, 3 \mathrm{H}) .{ }^{13} \mathrm{C} \mathrm{NMR}\left(100 \mathrm{MHz}, \mathrm{CDCl}_{3}\right) \delta(\mathrm{ppm}) 189.4$, 173.7, 164.2, 155.1, 152.7, 142.1, 131.4, 131.0, 129.5, 129.4, 122.8, 117.9, 111.1, 40.3, 32.4, 28.1, 17.6, 13.8. Elemental analysis: calcd (\%) for $\mathrm{C}_{20} \mathrm{H}_{22} \mathrm{~N}_{4} \mathrm{O}_{3}$ (366.41): C 65.56, H 6.05, found: C 65.87, H 6.35 .

5-(2-(1-Methylpyrrol-2-yl)phenyl)-1-pivalyltetrazole (20): Following the general procedure using 5-(2-bromophenyl)-1-pivalyltetrazole $\mathbf{1 b}(155 \mathrm{mg}, 0.5 \mathrm{mmol})$ and $\mathrm{N}$-methylpyrrole (162 $\mathrm{mg}, 2 \mathrm{mmol}$ ), the residue was purified by flash chromatography on silica gel (pentane- $\mathrm{Et}_{2} \mathrm{O}$, 70:30) to afford the desired compound $20(148 \mathrm{mg}, 96 \%)$ as a red solid $\left(\mathrm{mp}=80-83{ }^{\circ} \mathrm{C}\right) .{ }^{1} \mathrm{H}$ NMR $\left(400 \mathrm{MHz}, \mathrm{CDCl}_{3}\right) \delta(\mathrm{ppm}) 8.13(\mathrm{dd}, J=1.7$ and $7.3 \mathrm{~Hz}, 1 \mathrm{H}), 7.60-7.45(\mathrm{~m}, 3 \mathrm{H}), 6.67$ (dd, $J=1.8$ and $2.7 \mathrm{~Hz}, 1 \mathrm{H}), 6.18(\mathrm{dd}, J=2.7$ and $3.6 \mathrm{~Hz}, 1 \mathrm{H}), 6.07(\mathrm{dd}, J=1.8$ and $3.6 \mathrm{~Hz}$, 1H), $3.29(\mathrm{~s}, 3 \mathrm{H}), 1.29(\mathrm{~s}, 9 \mathrm{H}) .{ }^{13} \mathrm{C}$ NMR $\left(100 \mathrm{MHz}, \mathrm{CDCl}_{3}\right) \delta(\mathrm{ppm}) 173.5,164.9,132.8$, $132.5,131.7,130.9,129.6,128.3,125.1,122.4,109.1,107.9,33.9,32.3,27.9$. Elemental analysis: calcd (\%) for $\mathrm{C}_{17} \mathrm{H}_{19} \mathrm{~N}_{5} \mathrm{O}$ (309.37): C 66.00, H 6.19, found: C 66.21, H 6.00.

1-Pivalyl-5-(2',3',5',6'-tetrafluoro-[1,1'-biphenyl]-2-yl)tetrazole (21): Following the general procedure using 5-(2-bromophenyl)-1-pivalyltetrazole $\mathbf{1 b}(155 \mathrm{mg}, 0.5 \mathrm{mmol})$ and 1,2,3,5-tetrafluorobenzene $(113 \mathrm{mg}, 0.75 \mathrm{mmol})$, the residue was purified by flash chromatography on silica gel (pentane-Et ${ }_{2} \mathrm{O}, 70: 30$ ) to afford the desired compound 21 (127 $\mathrm{mg}, 67 \%)$ as a pale yellow oil. ${ }^{1} \mathrm{H}$ NMR $\left(400 \mathrm{MHz}, \mathrm{CDCl}_{3}\right) \delta(\mathrm{ppm}) 8.31-8.17(\mathrm{~m}, 1 \mathrm{H})$, $7.74-7.56(\mathrm{~m}, 2 \mathrm{H}), 7.44(\mathrm{dd}, J=3.1$ and $6.0 \mathrm{~Hz}, 1 \mathrm{H}), 7.15(\mathrm{tt}, J=7.3$ and $9.5 \mathrm{~Hz}, 1 \mathrm{H}), 1.34$ (s, 9H). ${ }^{13} \mathrm{C}$ NMR (100 MHz, $\left.\mathrm{CDCl}_{3}\right) \delta(\mathrm{ppm}) 173.1,163.5,145.8(\mathrm{dm}, J=250.1 \mathrm{~Hz}), 143.9$ $(\mathrm{dm}, J=250.1 \mathrm{~Hz}), 132.0,131.3,129.9,129.4,126.1,124.2,120.6(\mathrm{~d}, J=17.7 \mathrm{~Hz}), 105.6(\mathrm{t}$, $J=22.5 \mathrm{~Hz}$ ), 32.3, 27.9. Elemental analysis: calcd (\%) for $\mathrm{C}_{18} \mathrm{H}_{14} \mathrm{~F}_{4} \mathrm{~N}_{4} \mathrm{O}$ (378.32): C 57.14, H 3.73, found: C 57.31, H 4.02.

$2^{\prime}, 3^{\prime}, 5^{\prime}, 6^{\prime}-$ Tetrafluoro-2'"-(1-pivaloyltetrazol-5-yl)-[1,1':4',1''-terphenyl]-4-carbonitrile (22): Following the general procedure using 4-bromobenzonitrile (100 mg, $0.55 \mathrm{mmol})$ and 1 pivalyl-5-(2',3',5',6'-tetrafluoro-[1,1'-biphenyl]-2-yl)tetrazole 21 (189 $\mathrm{mg}, 0.5 \mathrm{mmol})$, the residue was purified by flash chromatography on silica gel (pentane-Et $2 \mathrm{O}, 80: 20$ ) to afford the desired compound $22(151 \mathrm{mg}, 63 \%)$ as a yellow solid $\left(\mathrm{mp}=207-209{ }^{\circ} \mathrm{C}\right) .{ }^{1} \mathrm{H}$ NMR $(400$ $\left.\mathrm{MHz}, \mathrm{CDCl}_{3}\right) \delta(\mathrm{ppm}) 8.31-8.17(\mathrm{~m}, 1 \mathrm{H}), 7.88-7.78(\mathrm{~m}, 2 \mathrm{H}), 7.75-7.64(\mathrm{~m}, 4 \mathrm{H}), 7.50$ $(\mathrm{dd}, J=3.2$ and $5.9 \mathrm{~Hz}, 1 \mathrm{H}), 1.40(\mathrm{~s}, 9 \mathrm{H}) .{ }^{13} \mathrm{C} \mathrm{NMR}\left(100 \mathrm{MHz}, \mathrm{CDCl}_{3}\right) \delta(\mathrm{ppm}) 173.1$, $163.3,144.5(\mathrm{dm}, J=251.1 \mathrm{~Hz}), 143.6(\mathrm{dm}, J=251.1 \mathrm{~Hz}), 132.4,132.2,132.0,131.3,131.0$, 130.1, 129.3, 125.8, 124.1, 120.3 (t, $J=16.4 \mathrm{~Hz}), 118.4$ (d, $J=15.0 \mathrm{~Hz}), 118.2,113.1,32.4$, 28.0. Elemental analysis: calcd (\%) for $\mathrm{C}_{25} \mathrm{H}_{17} \mathrm{~F}_{4} \mathrm{~N}_{5} \mathrm{O}$ (479.43): C 62.63, H 3.57; found: $\mathrm{C}$ 62.79, H 3.69 .

2-Ethyl-4-methyl-5-(2-(1-methyltetrazol-5-yl)phenyl)thiazole (23): Following the general procedure using 5-(2-bromophenyl)-1-methyltetrazole $1 \mathrm{c}(120 \mathrm{mg}, 0.5 \mathrm{mmol})$ and 2-ethyl-4methylthiazole $(95 \mathrm{mg}, 0.75 \mathrm{mmol}$ ), the residue was purified by flash chromatography on silica gel (pentane-Et $\left.\mathrm{E}_{2} \mathrm{O}, 70: 30\right)$ to afford the desired compound $23(127 \mathrm{mg}, 89 \%)$ as a pale yellow oil. ${ }^{1} \mathrm{H}$ NMR $\left(300 \mathrm{MHz}, \mathrm{CDCl}_{3}\right) \delta(\mathrm{ppm}) 8.02-7.91(\mathrm{~m}, 1 \mathrm{H}), 7.55-7.47(\mathrm{~m}, 2 \mathrm{H})$, $7.44(\mathrm{dd}, J=3.5$ and $5.8 \mathrm{~Hz}, 1 \mathrm{H}), 4.27(\mathrm{~s}, 3 \mathrm{H}), 2.99(\mathrm{q}, J=7.6 \mathrm{~Hz}, 2 \mathrm{H}), 2.02(\mathrm{~s}, 3 \mathrm{H}), 1.38$ (t, 
$J=7.6 \mathrm{~Hz}, 3 \mathrm{H}) .{ }^{13} \mathrm{C} \mathrm{NMR}\left(75 \mathrm{MHz}, \mathrm{CDCl}_{3}\right) \delta(\mathrm{ppm}) 170.9,164.7,148.4,132.5,131.5$, $130.1,129.8,128.7,128.4,128.1,39.4,26.8,15.3,14.3$. Elemental analysis: calcd (\%) for $\mathrm{C}_{14} \mathrm{H}_{15} \mathrm{~N}_{5} \mathrm{~S}$ (285.37): C 58.92, H 5.30; found: C 58.72, H 5.45.

2-Isobutyl-5-(2-(1-methyltetrazol-5-yl)phenyl)thiazole (24): Following the general procedure using 5-(2-bromophenyl)-1-methyltetrazole 1c $(120 \mathrm{mg}, 0.5 \mathrm{mmol})$ and 2isobutylthiazole (106 $\mathrm{mg}, 0.75 \mathrm{mmol}$ ), the residue was purified by flash chromatography on silica gel (pentane- $\left.\mathrm{Et}_{2} \mathrm{O}, 70: 30\right)$ to afford the desired compound 24 (139 $\mathrm{mg}, 93 \%$ ) as a pale yellow oil. ${ }^{1} \mathrm{H}$ NMR $\left(300 \mathrm{MHz}, \mathrm{CDCl}_{3}\right) \delta(\mathrm{ppm}) 7.86-7.77(\mathrm{~m}, 1 \mathrm{H}), 7.57-7.51(\mathrm{~m}, 2 \mathrm{H})$, $7.51-7.47(\mathrm{~m}, 1 \mathrm{H}), 7.45(\mathrm{~s}, 1 \mathrm{H}), 4.31(\mathrm{~s}, 3 \mathrm{H}), 2.84(\mathrm{~d}, J=7.2 \mathrm{~Hz}, 2 \mathrm{H}), 2.09(\mathrm{~m}, 1 \mathrm{H}), 1.00$ $(\mathrm{d}, J=6.8 \mathrm{~Hz}, 6 \mathrm{H}) .{ }^{13} \mathrm{C} \mathrm{NMR}\left(75 \mathrm{MHz}, \mathrm{CDCl}_{3}\right) \delta(\mathrm{ppm}) 170.7,164.7,140.8,135.6,131.5$, 131.2, 130.7, 130.1, 128.6, 127.1, 42.3, 39.4, 29.8, 22.2. Elemental analysis: calcd (\%) for $\mathrm{C}_{15} \mathrm{H}_{17} \mathrm{~N}_{5} \mathrm{~S}$ (299.39): C 60.18, H 5.72; found: C 60.11, H 5.49.

1-Methyl-5-(2-(5-pentylthiophen-2-yl)phenyl)tetrazole (25): Following the general procedure using 5-(2-bromophenyl)-1-methyltetrazole 1c $(120 \mathrm{mg}, 0.5 \mathrm{mmol})$ and 2- $n$ pentylthiophene $(116 \mathrm{mg}, 0.75 \mathrm{mmol})$, the residue was purified by flash chromatography on silica gel (pentane-Et $\left.{ }_{2} \mathrm{O}, 70: 30\right)$ to afford the desired compound $\mathbf{2 5}(127 \mathrm{mg}, 81 \%)$ as a pale yellow oil. ${ }^{1} \mathrm{H}$ NMR $\left(300 \mathrm{MHz}, \mathrm{CDCl}_{3}\right) \delta(\mathrm{ppm}) 7.68(\mathrm{dd}, J=1.6$ and $7.7 \mathrm{~Hz}, 1 \mathrm{H}), 7.59(\mathrm{dd}$, $J=1.5$ and $7.7 \mathrm{~Hz}, 1 \mathrm{H}), 7.51(\mathrm{dt}, J=1.6$ and $7.4 \mathrm{~Hz}, 1 \mathrm{H}), 7.42(\mathrm{dt}, J=1.5$ and $7.4 \mathrm{~Hz}, 1 \mathrm{H})$, $6.69(\mathrm{~d}, J=3.5 \mathrm{~Hz}, 1 \mathrm{H}), 6.64(\mathrm{~d}, J=3.5 \mathrm{~Hz}, 1 \mathrm{H}), 4.35(\mathrm{~s}, 3 \mathrm{H}), 2.83-2.70(\mathrm{~m}, 2 \mathrm{H}), 1.76-$ $1.58(\mathrm{~m}, 2 \mathrm{H}), 1.44-1.30(\mathrm{~m}, 4 \mathrm{H}), 0.92(\mathrm{t}, J=6.7 \mathrm{~Hz}, 3 \mathrm{H}),{ }^{13} \mathrm{C} \mathrm{NMR}\left(75 \mathrm{MHz}, \mathrm{CDCl}_{3}\right) \delta$ (ppm) 165.3, 146.8, 139.0, 135.1, 130.9, 130.9, 130.0, 127.5, 126.6, 126.4, 124.1, 39.4, 31.3, 30.0, 22.4, 14.0. Elemental analysis: calcd (\%) for $\mathrm{C}_{17} \mathrm{H}_{20} \mathrm{~N}_{4} \mathrm{~S}$ (312.43): C 65.35, H 6.45; found: C 65.49, H 6.72 .

1-Methyl-5-(2-(5-methylfuran-2-yl)phenyl)tetrazole (26): Following the general procedure using 5-(2-bromophenyl)-1-methyltetrazole 1c (120 mg, $0.5 \mathrm{mmol})$ and 2-methylfuran (62 $\mathrm{mg}, 0.75 \mathrm{mmol}$ ), the residue was purified by flash chromatography on silica gel (pentane$\left.\mathrm{Et}_{2} \mathrm{O}, 70: 30\right)$ to afford the desired compound $26(108 \mathrm{mg}, 90 \%)$ as a brown yellow oil. ${ }^{1} \mathrm{H}$ NMR $\left(300 \mathrm{MHz}, \mathrm{CDCl}_{3}\right) \delta(\mathrm{ppm}) 7.75(\mathrm{dd}, J=1.2$ and $7.5 \mathrm{~Hz}, 1 \mathrm{H}), 7.62(\mathrm{dd}, J=1.5$ and 7.5 $\mathrm{Hz}, 1 \mathrm{H}), 7.53(\mathrm{dt}, J=1.5$ and $7.7 \mathrm{~Hz}, 1 \mathrm{H}), 7.38(\mathrm{dt}, J=1.2$ and $7.5 \mathrm{~Hz}, 1 \mathrm{H}), 6.07(\mathrm{~d}, J=3.2$ $\mathrm{Hz}, 1 \mathrm{H}), 6.00-5.92(\mathrm{~m}, 1 \mathrm{H}), 4.41(\mathrm{~s}, 3 \mathrm{H}), 2.43-1.70(\mathrm{~m}, 3 \mathrm{H}) .{ }^{13} \mathrm{C} \mathrm{NMR}\left(75 \mathrm{MHz}, \mathrm{CDCl}_{3}\right)$ $\delta(\mathrm{ppm}) 165.6,152.1,150.4,131.1,131.1,130.1,127.8,127.1,124.3,109.7,107.5,39.4$, 13.6. Elemental analysis: calcd (\%) for $\mathrm{C}_{13} \mathrm{H}_{12} \mathrm{~N}_{4} \mathrm{O}$ (240.26): C 64.99, H 5.03; found: C 65.18, H 5.39.

1-(5-(2-(1-Methyltetrazol-5-yl)phenyl)furan-2-yl)propan-2-one (27): Following the general procedure using 5-(2-bromophenyl)-1-methyltetrazole $1 \mathrm{c}(120 \mathrm{mg}, 0.5 \mathrm{mmol})$ and 1(furan-2-yl)propan-2-one $(93 \mathrm{mg}, 0.75 \mathrm{mmol})$, the residue was purified by flash chromatography on silica gel (pentane- $\mathrm{Et}_{2} \mathrm{O}, 70: 30$ ) to afford the desired compound 27 (121 $\mathrm{mg}, 86 \%)$ as a brown yellow oil. ${ }^{1} \mathrm{H}$ NMR $\left(300 \mathrm{MHz}, \mathrm{CDCl}_{3}\right) \delta(\mathrm{ppm}) 7.76-7.64(\mathrm{~m}, 2 \mathrm{H})$, $7.54(\mathrm{dt}, J=1.5$ and $7.7 \mathrm{~Hz}, 1 \mathrm{H}), 7.43(\mathrm{dt}, J=1.4$ and $7.5 \mathrm{~Hz}, 1 \mathrm{H}), 6.30(\mathrm{~d}, J=3.3 \mathrm{~Hz}, 1 \mathrm{H})$, $6.22(\mathrm{~d}, J=3.3 \mathrm{~Hz}, 1 \mathrm{H}), 4.42(\mathrm{~s}, 3 \mathrm{H}), 3.61(\mathrm{~s}, 2 \mathrm{H}), 2.14(\mathrm{~s}, 3 \mathrm{H}) .{ }^{13} \mathrm{C}$ NMR $\left(75 \mathrm{MHz}, \mathrm{CDCl}_{3}\right)$ $\delta(\mathrm{ppm}) 203.8,165.3,152.3,148.2,131.1,130.6,130.1,128.3,127.8,125.0,110.1,109.6$, 43.3, 39.5, 29.2. Elemental analysis: calcd (\%) for $\mathrm{C}_{15} \mathrm{H}_{14} \mathrm{~N}_{4} \mathrm{O}_{2}$ (282.30): C 63.82, H 5.00; found: C 64.08, H 4.97.

1-Methyl-5-(2-(1-methylpyrrol-2-yl)phenyl)tetrazole (28): Following the general procedure using 5-(2-bromophenyl)-1-methyltetrazole 1c $(120 \mathrm{mg}, 0.5 \mathrm{mmol})$ and $\mathrm{N}$ methylpyrrole (162 mg, $2 \mathrm{mmol}$ ), the residue was purified by flash chromatography on silica gel (pentane-Et $\left.{ }_{2} \mathrm{O}, 70: 30\right)$ to afford the desired compound $\mathbf{2 8}(110 \mathrm{mg}, 92 \%)$ as a yellow solid 
$\left(\mathrm{mp}=99-101{ }^{\circ} \mathrm{C}\right) .{ }^{1} \mathrm{H}$ NMR $\left(400 \mathrm{MHz}, \mathrm{CDCl}_{3}\right) \delta(\mathrm{ppm}) 8.09-7.92(\mathrm{~m}, 1 \mathrm{H}), 7.63-7.41(\mathrm{~m}$, $3 \mathrm{H}), 6.67-6.64(\mathrm{~m}, 1 \mathrm{H}), 6.17(\mathrm{dd}, J=2.7$ and $3.5 \mathrm{~Hz}, 1 \mathrm{H}), 6.03(\mathrm{dd}, J=1.8$ and $3.5 \mathrm{~Hz}, 1 \mathrm{H})$, $4.29(\mathrm{~s}, 3 \mathrm{H}), 3.25(\mathrm{~s}, 3 \mathrm{H}) .{ }^{13} \mathrm{C}$ NMR $\left(100 \mathrm{MHz}, \mathrm{CDCl}_{3}\right) \delta(\mathrm{ppm}) 165.2,132.9,132.5,129.8$, $129.7,128.2,128.2,122.0,108.8,107.4,39.4,34.2$. Elemental analysis: calcd (\%) for $\mathrm{C}_{13} \mathrm{H}_{13} \mathrm{~N}_{5}$ (239.28): C 65.25, H 5.48; found: C 65.34, H 5.79.

1-Methyl-5-(2',3',4',5',6'-pentafluoro-[1,1'-biphenyl]-2-yl)tetrazole (29): Following the general procedure using 5-(2-bromophenyl)-1-methyltetrazole 1c $(120 \mathrm{mg}, 0.5 \mathrm{mmol})$ and pentafluorobenzene $(126 \mathrm{mg}, 0.75 \mathrm{mmol})$, the residue was purified by flash chromatography on silica gel (pentane-Et $\left.{ }_{2} \mathrm{O}, 70: 30\right)$ to afford the desired compound $29(113 \mathrm{mg}, 69 \%)$ as a yellow solid $\left(\mathrm{mp}=72-74{ }^{\circ} \mathrm{C}\right) .{ }^{1} \mathrm{H}$ NMR $\left(400 \mathrm{MHz}, \mathrm{CDCl}_{3}\right) \delta(\mathrm{ppm}) 8.35-8.21(\mathrm{~m}, 1 \mathrm{H})$, $7.71-7.54(\mathrm{~m}, 2 \mathrm{H}), 7.41(\mathrm{td}, J=1.9$ and $7.2 \mathrm{~Hz}, 1 \mathrm{H}), 4.31(\mathrm{~s}, 3 \mathrm{H}) .{ }^{13} \mathrm{C}$ NMR $(100 \mathrm{MHz}$, $\left.\mathrm{CDCl}_{3}\right) \delta(\mathrm{ppm}) 164.2,144.4(\mathrm{dm}, J=253.2 \mathrm{~Hz}), 140.9(\mathrm{dm}, J=253.2 \mathrm{~Hz}), 137.6(\mathrm{dm}, J=$ $253.2 \mathrm{~Hz}), 132.0,130.2,130.0,129.7,127.4,125.1,115.4(\mathrm{~m}), 39.5$. Elemental analysis: calcd (\%) for $\mathrm{C}_{14} \mathrm{H}_{7} \mathrm{~F}_{5} \mathrm{~N}_{4}$ (326.22): C 51.54, H 2.16; found: C 51.74, H 2.06.

1-Methyl-5-(2',3',5',6'-tetrafluoro-[1,1'-biphenyl]-2-yl)tetrazole (30): Following the general procedure using 5-(2-bromophenyl)-1-methyltetrazole $1 \mathrm{c}(120 \mathrm{mg}, 0.5 \mathrm{mmol})$ and 1,2,3,5-tetrafluorobenzene $(113 \mathrm{mg}, 0.75 \mathrm{mmol})$, the residue was purified by flash chromatography on silica gel (pentane- $\mathrm{Et}_{2} \mathrm{O}, 70: 30$ ) to afford the desired compound $\mathbf{3 0}$ (100 $\mathrm{mg}, 65 \%)$ as a yellow solid $\left(\mathrm{mp}=178-181{ }^{\circ} \mathrm{C}\right) .{ }^{1} \mathrm{H} \mathrm{NMR}\left(400 \mathrm{MHz}, \mathrm{CDCl}_{3}\right) \delta(\mathrm{ppm}) 8.26$ $(\mathrm{dd}, J=2.3$ and $7.4 \mathrm{~Hz}, 1 \mathrm{H}), 7.68-7.57(\mathrm{~m}, 2 \mathrm{H}), 7.44(\mathrm{dd}, J=2.0$ and $6.8 \mathrm{~Hz}, 1 \mathrm{H}), 7.11(\mathrm{tt}, J$ $=7.3$ and $9.7 \mathrm{~Hz}, 1 \mathrm{H}), 4.29(\mathrm{~s}, 3 \mathrm{H}) .{ }^{13} \mathrm{C} \mathrm{NMR}\left(100 \mathrm{MHz}, \mathrm{CDCl}_{3}\right) \delta(\mathrm{ppm}) 164.3,145.9(\mathrm{dm}$, $J=248.4 \mathrm{~Hz}), 144.0145 .9(\mathrm{dm}, J=248.4 \mathrm{~Hz}), 131.8,130.1,129.8,129.7,127.3,121.2(\mathrm{~d}, J$ $=22.7 \mathrm{~Hz}), 105.2(\mathrm{t}, J=22.5 \mathrm{~Hz}) 39.4$. Elemental analysis: calcd $(\%)$ for $\mathrm{C}_{14} \mathrm{H}_{8} \mathrm{~F}_{4} \mathrm{~N}_{4}$ (308.23): C 54.55, H 2.62, found: C 54.76, H 2.49.

2,6-Difluoro-2'-(1-methyltetrazol-5-yl)-[1,1'-biphenyl]-4-carbonitrile (31): Following the general procedure using 5-(2-bromophenyl)-1-methyltetrazole 1c $(120 \mathrm{mg}, 0.5 \mathrm{mmol})$ and 3,5-difluorobenzonitrile (104 $\mathrm{mg}, 0.75 \mathrm{mmol})$, the residue was purified by flash chromatography on silica gel (pentane- $\mathrm{Et}_{2} \mathrm{O}, 80: 20$ ) to afford the desired compound $\mathbf{3 1}$ (104 $\mathrm{mg}, 70 \%)$ as a yellow solid $\left(\mathrm{mp}=205-208{ }^{\circ} \mathrm{C}\right) .{ }^{1} \mathrm{H} \mathrm{NMR}\left(400 \mathrm{MHz}, \mathrm{CDCl}_{3}\right) \delta(\mathrm{ppm}) 8.33-$ $8.21(\mathrm{~m}, 1 \mathrm{H}), 7.70-7.56(\mathrm{~m}, 2 \mathrm{H}), 7.48-7.37(\mathrm{~m}, 1 \mathrm{H}), 7.31-7.21(\mathrm{~m}, 2 \mathrm{H}), 4.27(\mathrm{~s}, 3 \mathrm{H})$. ${ }^{13} \mathrm{C}$ NMR $\left(100 \mathrm{MHz}, \mathrm{CDCl}_{3}\right) \delta(\mathrm{ppm}) 164.3,160.3(\mathrm{dd}, J=8.7$ and $251.2 \mathrm{~Hz}), 131.5,130.1$, 129.9, 129.6, 127.1, 126.1, 124.1 (t, $J=20.6 \mathrm{~Hz}), 116.8(\mathrm{t}, J=3.6 \mathrm{~Hz}), 115.4(\mathrm{~d}, J=30.1$ $\mathrm{Hz}), 112.9(\mathrm{t}, J=11.9 \mathrm{~Hz}), 39.5$. Elemental analysis: calcd (\%) for $\mathrm{C}_{15} \mathrm{H}_{9} \mathrm{~F}_{2} \mathrm{~N}_{5}$ (297.26): C 60.61, H 3.05; found: C 60.46, H 3.08.

\section{REFERENCES}

[1] a) D. J. Carini, J. V. Duncia, P. E. Aldrich, A. T. Chiu, A. L. Johnson, M. E. Pierce, W. A. Price, J. B. Santella, G. J. Wells, J. Med. Chem. 1991, 34, 2525-2547; b) K. Kubo, Y. Kohara, Y. Yoshimura, Y. Inada, Y. Shibouta, Y. Furukawa, T. Kato, K. Nishikawa, T. Naka, J. Med. Chem. 1993, 36, 2343-2349; c) C. A. Bernhart, P. M. Perreaut, B. P. Ferrari, Y. A. Muneaux, J. L. A. Assens, J. Clement, F. Haudricourt, C. F. Muneaux, J. E. Taillades, J. Med. Chem. 1993, 36, 3371-3380; d) R. R. Wexler, W. J. Greenlee, J. D. Irvin, M. R. Goldberg, K. Prendergast, R. D. Smith, P. B. M. W. M. Timmermans, J. Med. Chem. 1996, 39, 625-656.

[2] L. Iouzalen, O. Stepien, P. Marche, J. Pharmacol. Exp. Ther. 1999, 289, 181-187.

[3] A. Alonen, M. Finel, R. Kostiainen, Biochem. Pharmacol. 2008, 76, 763-772.

[4] a) R. D. Larsen, A. O. King, C. Y. Chen, E. G. Corley, B. S. Foster, F. E. Roberts, C. Yang, D. R. Lieberman, R. A. Reamer, D. M. Tschaen, T. R. Verhoeven, P. J. Reider, Y. S. Lo, L. T. Rossano, A. S. Brookes, D. Meloni, J. R. Moore, J. F. Arnett, J. Org. Chem. 1994, 59, 6391-6394; b) R. Rai, K. B. Aubrecht, D. B. Collum, Tetrahedron Lett. 1995, 36, 3111-3114; c) N. Cousaert, P. Toto, N. Willand, B. Deprez, Tetrahedron Lett. 2005, 46, 6529-6532; d) N. Cousaert, N. Willand, J.-C. Gesquiere, A. 
Tartar, B. Deprez, R. Deprez-Poulain, Tetrahedron Lett. 2008, 49, 2743-2747; e) U. Beutler, M. Boehm, P. C. Fuenfschilling, T. Heinz, J.-P. Mutz, U. Onken, M. Mueller, W. Zaugg, Org. Process Res. Dev. 2007, 11, 892-898; f) S. K. N, S. B. Reddy, B. K. Sinha, K. Mukkanti, R. Dandala, Org. Process Res. Dev. 2009, 13, 1185-1189.

[5] a) F. Bellina, S. Cauteruccio, R. Rossi, Curr. Org. Chem. 2008, 12, 774-790; b) F. Kakiuchi, T. Kochi, Synthesis 2008, 3013-3039; c) L. Ackermann, R. Vicente, A. R. Kapdi, Angew. Chem. Int. Ed. 2009, 48, 9792-9826; d) F. Bellina, R. Rossi, Chem. Rev. 2009, 110, 1082-1146; e) F. Bellina, R. Rossi, Tetrahedron 2009, 65, 10269-10310; f) X. Chen, K. M. Engle, D.-H. Wang, J.-Q. Yu, Angew. Chem. Int. Ed. 2009, 48, 5094-5115; g) L. Joucla, L. Djakovitch, Adv. Synth. Catal. 2009, 351, 673-714; h) T. W. Lyons, M. S. Sanford, Chem. Rev. 2010, 110, 1147-1169; i) E. M. Beck, M. J. Gaunt, Top. Curr. Chem. 2010, 292, 85-121; j) T. Satoh, M. Miura, Synthesis 2010, 3395-3409; k) C.-L. Sun, B.-J. Li, Z.J. Shi, Chem. Commun. 2010, 46, 677-685; 1) S. H. Cho, J. Y. Kim, J. Kwak, S. Chang, Chem. Soc. Rev. 2011, 40, 5068-5083; m) N. Kuhl, M. N. Hopkinson, J. Wencel-Delord, F. Glorius, Angew. Chem. Int. Ed. 2012, 51, 10236-10254; n) B.-J. Li, Z.-J. Shi, Chem. Soc. Rev. 2012, 41, 5588-5598; o) M. C. White, Synlett 2012, 23, 2746-2748; p) S. I. Kozhushkov, L. Ackermann, Chem. Sci. 2013, 4, 886-896; q) H. Liu, G. Laurenczy, N. Yan, P. J. Dyson, Chem. Commun. 2014, 50, 341-343; r) R. Rossi, F. Bellina, M. Lessi, C. Manzini, Adv. Synth. Catal. 2014, 356, 17-117; s) M. Zhang, Y. Zhang, X. Jie, H. Zhao, G. Li, W. Su, Org. Chem. Front. 2014, 1, 843-895; t) K. Yuan, J.-F. Soulé, H. Doucet, ACS Catal. 2015, 5, 978-991; u) M. R. Yadav, R. K. Rit, M. Shankar, A. K. Sahoo, Asian J. Org. Chem. 2015, 4, 846-864; v) C. B. Bheeter, L. Chen, J.-F. Soulé, H. Doucet, Catal. Sci. Technol. 2016, 6, $2005-$ 2049.

[6] M. Seki, ACS Catal. 2011, 1, 607-610.

[7] M. Seki, M. Nagahama, J. Org. Chem. 2011, 76, 10198-10206.

[8] M. Seki, Synthesis 2012, 44, 3231-3237.

[9] E. Diers, N. Y. Phani Kumar, T. Mejuch, I. Marek, L. Ackermann, Tetrahedron 2013, 69, 4445-4453.

[10] R. A. Rivero, N. J. Kevin, E. E. Allen, Bioorg. Med. Chem. Lett. 1993, 3, 1119-1124.

[11] a) M. He, J.-F. Soulé, H. Doucet, ChemCatChem 2014, 6, 1824-1859; b) T. Yan, L. Zhao, M. He, J.-F. Soulé, C. Bruneau, H. Doucet, Adv. Synth. Catal. 2014, 356, 1586-1596; c) M. He, J.-F. Soulé, H. Doucet, ChemCatChem 2015, 7, 2130-2140.

[12] M. Lafrance, C. N. Rowley, T. K. Woo, K. Fagnou, J. Am. Chem. Soc. 2006, 128, 8754-8756.

[13] M. Seki, Synthesis 2014, 46, 3249-3255.

[14] C. Behloul, A. Chouti, D. Guijarro, C. Najera, M. Yus, Synthesis 2015, 47, 507-510.

[15] R. P. Singh, R. D. Verma, D. T. Meshri, J. n. M. Shreeve, Angew. Chem., Int. Ed. Engl. 2006, 45, 35843601.

[16] E. Vieira, J. Huwyler, S. Jolidon, F. Knoflach, V. Mutel, J. Wichmann, Bioorg. Med. Chem. Lett. 2005, $15,4628-4631$.

[17] R. S. Upadhayaya, S. Jain, N. Sinha, N. Kishore, R. Chandra, S. K. Arora, Eur. J. Med. Chem. 2004, 39, 579-592.

[18] T. Cantat, E. Génin, C. Giroud, G. Meyer, A. Jutand, J. Organomet. Chem. 2003, 687, 365-376. 


\section{Graphical Table of Contents}

Reactivity of $\mathrm{N}$-Protected 5-(2-Bromophenyl)tetrazoles in Palladium-Catalyzed Direct Arylation of Heteroarenes or Fluorobenzenes.

S. Chikhi, S. Djebbar, J.-F. Soulé, * H. Doucet*.

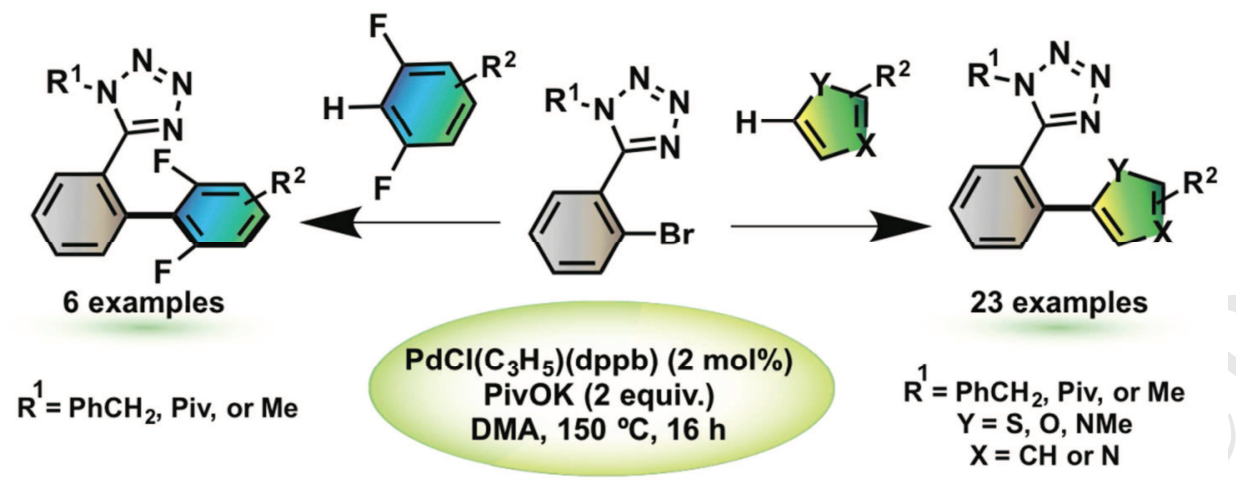

$\mathrm{N}$-protected 5-(2-bromophenyl)tetrazole derivatives have been used as aryl sources in $\mathrm{C}-\mathrm{H}$ bond arylation of a wide range of heteroarenes and some polyfluorobenzenes, using an airstable diphosphine palladium catalyst to afford (2-heteroarylphenyl)tetrazoles and fluorinated biphenyltetrazoles in high yields. 


\section{Highlights.}

- $\quad N$-protected 5-(2-bromophenyl)tetrazoles can be efficiently coupled heteroarenes

- The poisoning effect tetrazole was inhibited using $N$-alkyl or $N$-acyl substituents

- 30 novel (2-(hetero)arylphenyl)tetrazole derivatives were synthetized. 